\title{
SHAPE RECONSTRUCTIONS BY USING PLASMON RESONANCES
}

\author{
Ming-Hui Ding ${ }^{1}$, Hongyu Lid ${ }^{2, *}$ And Guang-Hui ZhenG ${ }^{1, *}$
}

\begin{abstract}
We study the shape reconstruction of an inclusion from the faraway measurement of the associated electric field. This is an inverse problem of practical importance in biomedical imaging and is known to be notoriously ill-posed. By incorporating Drude's model of the permittivity parameter, we propose a novel reconstruction scheme by using the plasmon resonance with a significantly enhanced resonant field. We conduct a delicate sensitivity analysis to establish a sharp relationship between the sensitivity of the reconstruction and the plasmon resonance. It is shown that when plasmon resonance occurs, the sensitivity functional blows up and hence ensures a more robust and effective construction. Then we combine the Tikhonov regularization with the Laplace approximation to solve the inverse problem, which is an organic hybridization of the deterministic and stochastic methods and can quickly calculate the minimizer while capture the uncertainty of the solution. We conduct extensive numerical experiments to illustrate the promising features of the proposed reconstruction scheme.
\end{abstract}

Mathematics Subject Classification. 65N21, 35R30, 78A46, 65H50.

Received November 23, 2021. Accepted February 14, 2022.

\section{INTRODUCTION}

Plasmon resonance is the resonant oscillation of conduction electrons at the interface between negative and positive permittivity material stimulated by incident field. Plasmonics is revolutionizing many light-based technologies via electron oscillations in metals. We refer to $[10,11,36,47,50,53]$ and the references cited therein for many striking optical, phononic, biomedical, diagnostic and therapeutic applications in the physical literature. Recent studies have revealed the deep and intriguing connection between the plasmon resonance and the spectral study of the Neumann-Poincaré operator $[2,6,7,12,23,24,31,42,45]$. In addition, there are many theoretical understandings and conceptual proposals about plasmonic devices.

In [5], by analyzing the imaginary part of the Green function, it is shown that one can achieve super-resolution and super-focusing by using plasmonic nanoparticles. In $[6,7,12]$, it is shown that the plasmon resonance concentrates and localises at high-curvature places, which can provide potential application in super-resolution imaging of plasmon particles. We would also like to mention in passing some related studies on plasmonic cloaking $[2,9,16,22,23,38-41,46,56]$.

Keywords and phrases. Shape reconstruction, plasmon resonance, sensitivity analysis, Tikhonov regularization, Laplace approximation.

1 School of Mathematics, Hunan University, Changsha 410082, P.R. China.

2 Department of Mathematics, City University of Hong Kong, Kowloon, Hong Kong, P.R. China.

*Corresponding author: hongyliu@cityu.edu.hk(Hongyu Liu); zhenggh2012@hnu.edu.cn(Guang-Hui Zheng) 
In this paper, we study the shape reconstruction of an inclusion from the faraway measurement of the associated electric field. This is an inverse problem of practical importance in biomedical imaging and is known to be notoriously ill-posed. By incorporating Drude's model of the permittivity parameter, we propose a novel reconstruction scheme by using the plasmon resonance with a significantly enhanced resonant field.

We next introduce the mathematical formulation of the inverse shape problem for our study. Let $D \subset \mathbb{R}^{2}$ be a bounded domain with a connected complement $\mathbb{R}^{2} \backslash \bar{D}$. Given a harmonic function $H$, we consider the following electrostatic problem:

$$
\left\{\begin{array}{l}
\nabla \cdot(\varepsilon \nabla u(x))=0 \quad \text { in } \mathbb{R}^{2} \\
u(x)-H(x)=O\left(|x|^{-1}\right) \text { as }|x| \rightarrow+\infty
\end{array}\right.
$$

where

$$
\varepsilon=\varepsilon_{D} \chi(D)+\varepsilon_{m} \chi\left(\mathbb{R}^{2} \backslash \bar{D}\right),
$$

and $\chi$ is the characteristic function. (1.1)-(1.2) describes the transverse electromagnetic propagation in the quasi-static regime. $u$ signifies the transverse electric field and $\varepsilon$ signifies the permittivity parameter of the medium. Throughout, we shall assume that the background parameter $\varepsilon_{m}$ is a positive constant, whereas $\varepsilon_{D}$ is a complex-valued function of the illuminating frequency and fulfils the Drude's model. We shall supply more details about the Drude model in what follows. The shape reconstruction problem can be formulated as follows:

Inverse Problem (IP): Identify the shape of the inclusion, namely $\partial D$, from the measurement data $u^{s}=u-H$ on $\partial \Omega$ with $D \Subset \Omega$ associated with a fixed incident field $H$. For simplicity, we take $\Omega$ to be a central ball of radius $r \in \mathbb{R}_{+}$with $r$ sufficiently large. Hence, the measurement represents the far-field pattern of the electric field.

The shape reconstruction problem introduced above is severely ill-posed and highly nonlinear. First, it is well known that due to the diffraction limit, the far field excited by the object carries information on a scale much larger than the operating wavelength, while information on a scale smaller than the operating wavelength is confined near the object itself. In addition, the scattering information in the quasi-static regime is very weak, and in the presence of measurement noise, the signal-to-noise ratio in the far field is low and signal distortion is serious [8]. We also refer to $[3,7,13-15,17,18,20,21,25,28,30,33,34,43,44,55]$ for related studies in the literature on this inverse shape problem.

In this article, we first perform a shape sensitivity analysis and derive the shape sensitivity functional with respect to domain perturbation by a delicate asymptotic analysis. We establish the spectral expansion of the shape sensitivity functional, from which we can conclude the sharp relationship between the reconstruction sensitivity and the plasmon resonance. It indicates that the plasmon resonant field can render a more robust and effective reconstruction. Moreover, in order to overcome the ill-posedness, we combine the Tikhonov regularization method with the Laplace approximation (LA) to solve the inverse problem. This hybrid method is essentially the organic combination of the deterministic method and stochastic method, which can rapidly calculate the minimizer (Maximum a posteriori estimation point (MAP)) and capture statistical information of the solution more effectively. To provide a global view of our study, the major contributions of this work can be summarised as follows.

1. By using the layer-potential perturbation technique, we rigorously derive the asymptotic expansion of the perturbed far field with respect to the shape perturbation. Furthermore, we obtain the representation formula of the shape sensitivity functional.

2. Based on the spectral theory of the Neumann-poincaré operator, we establish the delicate spectral expansion of the shape sensitivity functional. It indicates that when plasmon resonance occurs, the shape sensitivity can be improved dramatically.

3. Due to the severe ill-posedness of inverse problem, we use plasmon resonance to enhance the sensitivity, and then combine the Tikhonov regularization method with the Laplace approximation to solve the inverse 
problem and quantify the uncertainty of the solution. Compared with the standard method, our numerical results show that the proposed method can significantly improve the accuracy and robustness of the numerical reconstruction.

The rest of the paper is organized as follows. In Section 2, we provide preliminary knowledge on layer potential operators and plasmon resonance. In Section 3, we conduct the sensitivity analysis for the perturbed domain, and derive the spectral expansion of the shape sensitivity functional. In Section 4, we discuss the combination of the Tikhonov regularization method and the Laplace approximation. Sections 5 and 6 are respectively devoted to numerical experiments and conclusion.

\section{Preliminaries}

\subsection{Layer potentials and Neumann-Poincaré operator}

We collect a number of preliminary results on the layer potentials, in particular the Neumann-Poincaré operator for our subsequent use. Throughout this paper, we consider a domain $D$ with a $C^{2}$ boundary. The $L^{2}$ inner product and the corresponding norm on $\partial D$ are denoted by $\langle\cdot, \cdot\rangle$ and $\|\cdot\|$ in short, respectively. The single layer potential $\mathcal{S}_{D}$ and double layer potential $\mathcal{D}_{D}$ associated with $D$ are given by

$$
\begin{aligned}
& \mathcal{S}_{D}[\varphi](x):=\int_{\partial D} \Gamma(x, y) \varphi(y) d \sigma(y), \quad x \in \mathbb{R}^{2}, \\
& \mathcal{D}_{D}[\varphi](x):=\int_{\partial D} \frac{\partial \Gamma(x, y)}{\partial \nu(y)} \varphi(y) d \sigma(y), \quad x \in \mathbb{R}^{2} \backslash \partial D,
\end{aligned}
$$

where $\varphi \in L^{2}(\partial D)$ is the density function, and the Green function $\Gamma(x, y)$ to the Laplacian in $\mathbb{R}^{2}$ is given by

$$
\Gamma(x, y)=\frac{1}{2 \pi} \ln |x-y| \text {. }
$$

The notations $\left.u\right|_{ \pm}$and $\left.\frac{\partial u}{\partial \nu}\right|_{ \pm}$denote the traces on $\partial D$ from the outside and inside of $D$, respectively. The following jump relations hold $[2,3]$ :

$$
\left.\frac{\partial \mathcal{S}_{D}[\varphi]}{\partial \nu}\right|_{ \pm}(x)=\left( \pm \frac{1}{2} I+\mathcal{K}_{D}^{*}\right)[\varphi](x), \quad x \in \partial D
$$

where $\mathcal{K}_{D}^{*}$ is known as the Neumann-Poincaré (NP) operator defined by

$$
\mathcal{K}_{D}^{*}[\varphi](x)=\frac{1}{2 \pi} \int_{\partial D} \frac{\langle x-y, \nu(x)\rangle}{|x-y|^{2}} \varphi(y) d \sigma(y) .
$$

Next, we recall some useful facts about the NP operator $\mathcal{K}_{D}^{*}[2,23,45]$.

Lemma 1. (i) The Calderón identity holds: $\mathcal{S}_{D} \mathcal{K}_{D}^{*}=\mathcal{K}_{D} \mathcal{S}_{D}$ on $H_{0}^{-\frac{1}{2}}$, where $H_{0}^{-\frac{1}{2}}$ is the zero mean subspace of $H^{-\frac{1}{2}}$;

(ii) The operator $\mathcal{K}_{D}^{*}$ is compact and self-adjoint in the Hilbert space $H_{0}^{-\frac{1}{2}}$ equipped with the following inner product

$$
\langle\varphi, \psi\rangle_{\mathcal{H}^{*}(\partial D)}=-\left\langle\mathcal{S}_{D}[\psi], \varphi\right\rangle_{\frac{1}{2},-\frac{1}{2}}
$$

with $\langle\cdot, \cdot\rangle_{-\frac{1}{2}, \frac{1}{2}}$ being the duality pairing between $H^{-1 / 2}(\partial D)$ and $H^{1 / 2}(\partial D)$

(iii) Let $\mathcal{H}^{*}(\partial D)$ be the space $H_{0}^{-\frac{1}{2}}(\partial D)$ with the new inner product in (ii). Let $\left(\lambda_{j}, \varphi_{j}\right), j=0,1,2, \ldots$ be the eigenvalue and normalized eigenfunction pair of $\mathcal{K}_{D}^{*}$ in $\mathcal{H}^{*}(\partial D)$, then $\lambda_{j} \in\left(-\frac{1}{2}, \frac{1}{2}\right)$ and $\lambda_{j} \rightarrow 0$ as $j \rightarrow \infty$;

(iv) The following representation formula holds: for any $\varphi \in \mathcal{H}^{*}(\partial D)$,

$$
\mathcal{K}_{D}^{*}[\varphi]=\sum_{j=1}^{\infty} \lambda_{j}\left\langle\varphi, \varphi_{j}\right\rangle_{\mathcal{H}^{*}(\partial D)} \varphi_{j}
$$




\subsection{Plasmon resonance}

We next briefly discuss the mathematical framework of plasmon resonance. We first give the form of the solution of equation (1.1). From [2,8,23], we have

$$
u(x)=H(x)+\mathcal{S}_{D}[\phi](x), \quad \text { for } x \in \mathbb{R}^{2},
$$

where $\phi \in L_{0}^{2}(\partial D):=\left\{\phi \in L^{2}(\partial D) ; \int_{\partial D} \phi=0\right\}$ satisfies

$$
\left(\lambda I-\mathcal{K}_{D}^{*}\right)[\phi](x)=\left.\frac{\partial H}{\partial \nu}\right|_{\partial D}, \quad x \in \partial D
$$

with $\lambda$ given by

$$
\lambda=\frac{\varepsilon_{D}+\varepsilon_{m}}{2\left(\varepsilon_{D}-\varepsilon_{m}\right)} .
$$

The permittivities of plasmon materials, such as noble metals, are different from the ordinary materials and may possess negative real parts. In fact, the electric permittivity $\varepsilon_{D}$ of the plasmon material is changing with respect to the operating frequency $\omega$. The $\varepsilon_{D}$ can be described by the Drude's model (see $\left.[4,27,51]\right)$,

$$
\varepsilon_{D}=\varepsilon_{D}(\omega)=\varepsilon_{0}\left(1-\frac{\omega_{p}^{2}}{\omega(\omega+i \gamma)}\right),
$$

where $\varepsilon_{0}$ is the electric permittivity of the vacuum and is given by $\varepsilon_{0}=9 \times 10^{-12} \mathrm{~F} / \mathrm{m}$. The plasmon frequency $\omega_{p}$ and the damping parameter $\gamma$ are strictly positive. When $\omega<\omega_{p}$ the real part of $\varepsilon_{D}$ can be negative. In particular, for the gold, the value of the plasmon frequency and the damping parameter are $\omega_{p}=2 \times 10^{15} s^{-1}$ and $\gamma=10^{14} s$, respectively. Let the operating frequency $\omega=6 \times 10^{14} \mathrm{~Hz}$ (visible light frequency), and from Drude's model (2.4), the electric permittivity of gold nanoparticle is calculated as $\varepsilon_{D} \approx(-9.8108+1.8018 i) \varepsilon_{0}$.

Now by applying the spectral decomposition of the $\mathcal{K}_{D}^{*}$ to the integral equation $(2.2)$, the density $\phi \in L_{0}^{2}(\partial D)$ becomes

$$
\phi=\sum_{j=1}^{\infty} \frac{\left\langle\frac{\partial H}{\partial \nu}, \varphi_{j}\right\rangle_{\mathcal{H}^{*}(\partial D)}}{\lambda-\lambda_{j}} \varphi_{j},
$$

where $\lambda_{j}$ are eigenvalues of $\mathcal{K}_{D}^{*}$ and they satisfy $\left|\lambda_{j}\right|<\frac{1}{2}$. When the real part of $\varepsilon_{D}(\omega)$ is negative, it holds that $\left|\mathfrak{R e}(\lambda(\omega))<\frac{1}{2}\right|$, where and also in what follows $\mathfrak{R e}(\lambda(\omega))$ signifies the real part of $\lambda(\omega)$. The frequency $\omega$ is called a plasmon resonance frequency if it satisfies

$$
\mathfrak{R e}(\lambda(\omega))=\lambda_{j},
$$

for some $j$, where $\lambda_{j}$ is an eigenvalue of the NP operator $\mathcal{K}_{D}^{*}$. For this reason, (2.6) is called the plasmon resonance condition. It is emphasized that only when the imaginary part $\mathfrak{I m}(\lambda(\omega))=0$ (lossless metal), the resonance condition (2.6) can imply the density $\phi$ in (2.5) blow up.

In (2.5) the density $\phi$ will be amplified when the plasmon resonance condition is reached provided that $\left\langle\frac{\partial H}{\partial \nu}, \varphi_{j}\right\rangle_{\mathcal{H}^{*}(\partial D)}$ is nonzero. As a result, the $j$-th mode of the far field $u-H$ will show a resonant behavior, and it is called that plasmon resonance occurs (see also [23]). As an illustration, we consider the cylindrical metal nanorod as an example. When the external electric field lines are parallel to the circular cross-section of the cylindrical metal nanorod, the physical process can be described by the two-dimensional mathematical model (1.1)-(1.2). Next, we set $\varepsilon_{m}=\varepsilon_{0}$ (vacuum) and notice that, as $D$ is a disk, the spectrum of $\mathcal{K}_{D}^{*}$ are $\left\{0, \frac{1}{2}\right\}$. If $\lambda_{j}=0$, from the plasmon resonance condition (2.6) and the fact that $\gamma$ is sufficiently small in (2.4), we nearly get $\mathfrak{R e}\left(\varepsilon_{D}(\omega)\right)=-\varepsilon_{m}$. This relationship is called the Fröhlich condition (see [48]). Furthermore, by Drude's model (2.4), we can obtain the resonance frequency $\omega=\sqrt{\frac{\omega_{p}^{2}}{2}-\gamma^{2}}$. When $\gamma=0$ (lossless metal), the resonance frequency $\omega=\frac{\omega_{p}}{\sqrt{2}}$ or $f=\frac{\omega_{p}}{2 \pi \sqrt{2}}$, which is called the Fröhlich frequency (see [51]). 


\section{Shape SENSITIVITY ANALYSIS}

\subsection{Sensitivity analysis for the perturbed domain}

We consider the sensitivity analysis for the shape reconstruction problem with a perturbed domain, namely evaluating the effect of the domain variations on the far-field measurement data.

Define the forward operator $\mathcal{F}: X \rightarrow H^{\frac{1}{2}}(\partial \Omega)$ on a subset $X \subset C^{2}(\partial D)$ :

$$
\mathcal{F}(\partial D)=\left.u^{s}\right|_{\partial \Omega}, \quad x \in X,
$$

where $\left.u^{s}\right|_{\partial \Omega}=\left.(u-H)\right|_{\partial \Omega}$ is the far-field measurement associated with (1.1) on the boundary $\partial \Omega$.

For a small $\epsilon \in \mathbb{R}_{+}$, we let $\partial D_{\epsilon}$ be an $\epsilon$-perturbation of $D$, i.e.,

$$
\partial D_{\epsilon}:=\{\tilde{x}=x+\epsilon h(x) \nu(x), x \in \partial D\},
$$

where $h \in C^{1}(\partial D)$, and $\nu$ is the outward unit normal vector to $\partial D$. The solution $u_{\epsilon}$ to (1.1) with $D_{\epsilon}$ has the following representation formula

$$
u_{\epsilon}=H(x)+\mathcal{S}_{D_{\epsilon}}\left[\tilde{\phi}_{\epsilon}\right](x), \quad x \in \mathbb{R}^{2},
$$

where the density function $\tilde{\phi}_{\epsilon}$ is the solution to

$$
\left(\lambda I-\mathcal{K}_{D_{\epsilon}}^{*}\right)\left[\tilde{\phi}_{\epsilon}\right](\tilde{x})=\left.\frac{\partial H}{\partial \tilde{\nu}}\right|_{\partial D_{\epsilon}} \quad \tilde{x} \in \partial D_{\epsilon}
$$

Let $\Psi_{\epsilon}$ be the diffeomorphism from $\partial D$ to $\partial D_{\epsilon}$ given by

$$
\Psi_{\epsilon}(x)=x+\epsilon h(x) \nu(x), \quad x \in \partial D .
$$

Moreover, we denote $\tilde{\nu}$ the outward unit normal vector to $\partial D_{\epsilon}$ and $d \tilde{\sigma}$ the line element of $\partial D_{\epsilon}$. The following expansions of $\tilde{\nu}$ and $d \tilde{\sigma}$ hold [1]:

$$
\begin{aligned}
\tilde{\nu}(\tilde{x}) & =\nu(x)-\epsilon h^{\prime}(x) T(x)+O\left(\epsilon^{2}\right), \\
d \tilde{\sigma}(\tilde{x}) & =d \sigma(x)-\epsilon \tau(x) h(x) d \sigma(x)+O\left(\epsilon^{2}\right) .
\end{aligned}
$$

Here and throughout the rest of the paper, $\tau(x)$ signifies the curvature of $\partial D$ at $x, T$ is the unit tangential vector to $\partial D$, and $h^{\prime}$ is the tangential derivative of $h$ on $\partial D$, i.e., $h^{\prime}=\frac{\partial h}{\partial T}$, where $\frac{\partial}{\partial \tilde{\nu}}$ denotes the outward normal derivative on $\partial D_{\epsilon}$.

In view of (3.1) and (3.2), in order to obtain the asymptotic formula of the perturbed far field $\left.u_{\epsilon}^{s}\right|_{\partial \Omega}:=\mathcal{F}\left(\partial D_{\epsilon}\right)$, we need to obtain the corresponding asymptotic expansions of the operators $\mathcal{K}_{D_{\epsilon}}^{*}$ and $\tilde{\phi}_{\epsilon}$. The following lemmas can be found in [1].

Lemma 2. For $\tilde{\phi} \in L^{2}\left(\partial D_{\epsilon}\right)$, let $\phi:=\tilde{\phi} \circ \Psi_{\epsilon}$. Then there exists a constant $C$ depending only on the $C^{2}$-norm of $\partial D$ and $\|h\|_{C^{1}}$ such that

$$
\left\|\left(\mathcal{K}_{D_{\epsilon}}^{*}[\tilde{\phi}]\right) \circ \Psi_{\epsilon}-\mathcal{K}_{D}^{*}[\phi]-\epsilon \mathcal{K}_{D}^{(1)}[\phi]\right\|_{L^{2}(\partial D)} \leq C \epsilon^{2}\|\phi\|_{L^{2}(\partial D)},
$$

with the operator $\mathcal{K}_{D}^{(1)}$ defined for any $\phi \in L^{2}(\partial D)$ by

$$
\mathcal{K}_{D}^{(1)}[\phi](x)=\text { p.v. } \int_{\partial D} K_{1}(x, y) \phi(y) d \sigma(y), \quad x \in \partial D,
$$


where

$$
\begin{aligned}
K_{1}(x, y) & =-2 \frac{\langle x-y, \nu(x)\rangle\langle x-y, h(x) \nu(x)-h(y) \nu(y)\rangle}{|x-y|^{4}} \\
& +\frac{\langle h(x) \nu(x)-h(y) \nu(y), \nu(x)\rangle}{|x-y|^{2}}-\frac{\left\langle x-y, \tau(x) h(x) \nu(x)+h^{\prime}(x) T(x)\right\rangle}{|x-y|^{2}} \\
& +\frac{\langle x-y, \nu(x)\rangle}{|x-y|^{2}}(h(x) \tau(x)-h(y) \nu(y)) .
\end{aligned}
$$

Here, p.v. stands for the Cauchy principal value.

In fact, we can rewrite the operator $\mathcal{K}_{D}^{(1)}$ in terms of more familiar operators as follows, , then we get

$$
\mathcal{K}_{D}^{(1)}[\phi]=-\frac{\partial}{\partial T}\left(h \frac{\partial \mathcal{S}_{D}[\phi]}{\partial T}\right)+\frac{\partial \mathcal{D}_{D}[h \phi]}{\partial \nu}+h \tau \mathcal{K}_{D}^{*}[\phi]-\mathcal{K}_{D}^{*}[h \tau \phi]
$$

Lemma 3. Let $\tilde{\phi}_{\epsilon}=\left(\lambda I-\mathcal{K}_{D_{\epsilon}}^{*}\right)^{-1}[\tilde{\nu} \cdot \nabla H], \phi_{\epsilon}=\tilde{\phi}_{\epsilon} \circ \Psi_{\epsilon}$, and $\phi=\left(\lambda I-\mathcal{K}_{D}^{*}\right)^{-1}[\nu \cdot \nabla H]$. Then we have

$$
\left\|\phi_{\epsilon}-\phi-\epsilon \phi^{(1)}\right\|_{L^{2}(\partial D)} \leq C \epsilon^{2}\|\phi\|_{L^{2}(\partial D)},
$$

where $C$ is a constant depending only on the $C^{2}$-norm of $\partial D$ and $\|h\|_{C^{1}}$ and

$$
\phi^{(1)}=\left(\lambda I-\mathcal{K}_{D}^{*}\right)^{-1}\left(h\left\langle\left(\nabla^{2} H\right) \nu, \nu\right\rangle-h^{\prime}\langle\nabla H, T\rangle+\mathcal{K}_{D}^{(1)} \phi\right) .
$$

Furthermore, for $\frac{\partial H}{\partial \tilde{\nu}}$ of (3.2), we can further obtain by using (3.3), that

$$
\frac{\partial H}{\partial \tilde{\nu}}=\nabla H(\Psi(x)) \cdot \tilde{\nu}(\Psi(x))=\frac{\partial H}{\partial \nu}(x)+\epsilon G^{(1)}(x)+O\left(\epsilon^{2}\right), \quad x \in \partial D
$$

where

$$
G^{(1)}=h(x)\left\langle\nabla^{2} H(x) \nu(x), \nu(x)\right\rangle-h^{\prime}(x)\langle\nabla H(x), T(x)\rangle .
$$

The asymptotic expansion of $\mathcal{F}\left(\partial D_{\epsilon}\right)$ under small perturbations of the boundary $\partial D$ in terms of the asymptotic parameter $\epsilon$ is given in the following theorem. We would like to point out that its proof is adapted from those in $[1,29]$.

Theorem 4. Suppose that $\phi, \phi_{d}$ satisfy

$$
\begin{aligned}
\left(\lambda I-\mathcal{K}_{D}^{*}\right)[\phi](y) & =\left.\frac{\partial H}{\partial \nu}\right|_{\partial D}, \\
\left(\lambda I-\mathcal{K}_{D}\right)\left[\phi_{d}\right](x, y) & =\Gamma(x, y), \quad x \in \partial \Omega, y \in \partial D,
\end{aligned}
$$

the following asymptotic expansion holds:

$$
\left.u_{\epsilon}^{s}(x)\right|_{\partial \Omega}-\left.u^{s}(x)\right|_{\partial \Omega}=\epsilon\langle h(y), P(x, y)\rangle_{L^{2}(\partial D)}+O\left(\epsilon^{2}\right), \quad x \in \partial \Omega, y \in \partial D,
$$

where

$$
P(x, y)=\frac{\partial \phi_{d}(x, y)}{\partial T(y)}\left(\frac{\partial\left(H(y)+\mathcal{S}_{D}[\phi](y)\right)}{\partial T(y)}\right)+\left(\frac{\partial\left(\mathcal{D}_{D}\left[\phi_{d}\right](x, y)+\Gamma(x, y)\right)}{\partial \nu(y)}\right) \phi(y) .
$$


Proof. From the form of the solution (3.1), it holds that

$$
\mathcal{F}\left(\partial D_{\epsilon}\right)=\int_{\partial D_{\epsilon}} \Gamma(x, \tilde{y}) \tilde{\phi}_{\epsilon}(\tilde{y}) d \tilde{\sigma}(\tilde{y}), x \in \partial \Omega
$$

where $\tilde{\phi}_{\epsilon}$ is solution to $(3.2), d \tilde{\sigma}(\tilde{y})$ has an expansion as $(3.4)$, and $\Gamma(x, \tilde{y})$ has the Taylor expansion as follows

$$
\Gamma(x, y+\epsilon h(y) \nu(y))=\Gamma(x, y)+\epsilon h(y) \frac{\partial \Gamma(x, y)}{\partial \nu(y)}+O\left(\epsilon^{2}\right) \quad y \in \partial D, x \in \partial \Omega .
$$

By virtue of Lemma 3, we can obtain that

$$
\begin{aligned}
\mathcal{F}\left(\partial D_{\epsilon}\right)-\mathcal{F}(\partial D)= & \epsilon \int_{\partial D} \Gamma(x, y) \phi^{(1)}(y) d \sigma(y)+\epsilon \int_{\partial D}\left(\frac{\partial \Gamma(x, y)}{\partial \nu(y)}-\tau(y) \Gamma(x, y)\right) \phi(y) h(y) d \sigma(y) \\
& +O\left(\epsilon^{2}\right) .
\end{aligned}
$$

Next, we first calculate the term $\int_{\partial D} \Gamma(x, y) \phi^{(1)}(y) d \sigma(y)$. From (3.7) and (3.11) we have

$$
\begin{aligned}
\int_{\partial D} \Gamma(x, y) \phi^{(1)}(y) d \sigma(y)= & \int_{\partial D}\left(\lambda I-\mathcal{K}_{D}\right) \phi_{d}(x, y) \phi^{(1)}(y) d \sigma(y) \\
= & \int_{\partial D} \phi_{d}(x, y)\left(\lambda I-\mathcal{K}_{D}^{*}\right) \phi^{(1)}(y) d \sigma(y) \\
= & \int_{\partial D} \phi_{d}(x, y)\left(h(y)\left\langle\left(\nabla^{2} H(y)\right) \nu(y), \nu(y)\right\rangle-h^{\prime}(y) \frac{\partial H(y)}{\partial T(y)}\right) d \sigma(y) \\
& +\int_{\partial D} \phi_{d}(x, y) \mathcal{K}_{D}^{(1)}[\phi](y) d \sigma(y) \\
= & : l_{1}+l_{2} .
\end{aligned}
$$

We treat $l_{1}, l_{2}$ separately. Since

$$
\begin{aligned}
\frac{\partial}{\partial T(y)}\left(\phi_{d}(x, y) \frac{\partial H(y)}{\partial T(y)}\right) & =\frac{\partial \phi_{d}(x, y)}{\partial T(y)} \frac{\partial H(y)}{\partial T(y)}+\phi_{d}(x, y)\left\langle\left(\nabla^{2} H(y)\right) T(y), T(y)\right\rangle \\
& +\phi_{d}(x, y) \tau(y) \frac{\partial H(y)}{\partial \nu(y)}
\end{aligned}
$$

and noting that $H(y)$ is a harmonic function and (3.13), $l_{1}$ has the following form

$$
\begin{aligned}
l_{1}= & \int_{\partial D} \phi_{d}(x, y)\left(h(y)\left\langle\left(\nabla^{2} H(y)\right) \nu(y), \nu(y)\right\rangle-h^{\prime}(y)\langle\nabla H(y), T(y)\rangle\right) d \sigma(y) \\
= & \int_{\partial D} h(y)\left(\phi_{d}(x, y)\left\langle\left(\nabla^{2} H(y)\right) \nu(y), \nu(y)\right\rangle+\frac{\partial \phi_{d}(x, y)}{\partial T(y)} \frac{\partial H(y)}{\partial T(y)}\right. \\
& \left.+\phi_{d}(x, y)\left\langle\left(\nabla^{2} H(y)\right) T(y), T(y)\right\rangle+\phi_{d}(x, y) \tau(y) \frac{\partial H(y)}{\partial \nu(y)}\right) d \sigma(y) \\
= & \int_{\partial D} h(y)\left(\frac{\partial \phi_{d}(x, y)}{\partial T(y)} \frac{\partial H(y)}{\partial T(y)}+\phi_{d}(x, y) \Delta H(y)+\phi_{d}(x, y) \tau(y) \frac{\partial H(y)}{\partial \nu(y)}\right) d \sigma(y) \\
= & \int_{\partial D} h(y)\left(\frac{\partial \phi_{d}(x, y)}{\partial T(y)} \frac{\partial H(y)}{\partial T(y)}+\phi_{d}(x, y) \tau(y) \frac{\partial H(y)}{\partial \nu(y)}\right) d \sigma(y) .
\end{aligned}
$$

Next, by (3.6), we have

$$
l_{2}=\int_{\partial D} \phi_{d}(x, y) \mathcal{K}_{D}^{(1)}[\phi](y) d \sigma(y)
$$




$$
\begin{aligned}
= & \int_{\partial D} \phi_{d}(x, y)\left[-\frac{\partial}{\partial T(y)}\left(h(y) \frac{\partial \mathcal{S}_{D}[\phi](y)}{\partial T(y)}\right)+\frac{\partial}{\partial \nu(y)} \mathcal{D}_{D}[\phi h](y)+h(y) \tau(y) \mathcal{K}_{D}^{*}[\phi](y)\right. \\
& \left.-\mathcal{K}_{D}^{*}[h \tau \phi](y)\right] d \sigma(y) \\
= & \int_{\partial D} h(y)\left[\frac{\partial \phi_{d}(x, y)}{\partial T(y)} \frac{\partial\left(\mathcal{S}_{D} \phi(y)\right)}{\partial T(y)}+\frac{\partial}{\partial \nu(y)} \mathcal{D}_{D}\left[\phi_{d}\right](x, y) \phi(y)+\phi_{d}(x, y) \tau(y) \mathcal{K}_{D}^{*}[\phi](y)\right. \\
& \left.-\tau(y) \phi(y) \mathcal{K}_{D}\left[\phi_{d}\right](x, y)\right] d \sigma(y),
\end{aligned}
$$

from which, together with the use of Proposition 4.1 in [1], we can further show that

$$
\int_{\partial D} \phi_{d}(x, y) \frac{\partial \mathcal{D}_{D}[h \phi](y)}{\partial \nu(y)} d \sigma(y)=\int_{\partial D} \frac{\partial \mathcal{D}_{D}\left[\phi_{d}\right](x, y)}{\partial \nu(y)} h(y) \phi(y) d \sigma(y) .
$$

Thus we obtain that

$$
\begin{aligned}
\mathcal{F}\left(\partial D_{\epsilon}\right)-\mathcal{F}(\partial D) & =\int_{\partial D} h(y)\left[\frac{\partial \phi_{d}(x, y)}{\partial T(y)}\left(\frac{\partial\left(\mathcal{S}_{D}[\phi]\right)(y)}{\partial T(y)}\right)+\frac{\partial\left(\mathcal{D}_{D}\left[\phi_{d}\right]\right)(x, y)}{\partial \nu(y)} \phi(y)\right. \\
& +\tau(y) \phi_{d}(x, y) \mathcal{K}_{D}^{*}[\phi](y)-\tau(y) \mathcal{K}_{D}\left[\phi_{d}\right](x, y) \phi(y) \\
& +\phi_{d}(x, y) \tau(y) \frac{\partial H(y)}{\partial \nu(y)}+\frac{\partial \phi_{d}(x, y)}{\partial T(y)} \frac{\partial H(y)}{\partial T(y)} \\
& \left.+\left(\frac{\partial \Gamma(x, y)}{\partial \nu(y)}-\tau(y) \Gamma(x, y)\right) \phi(y)\right] d \sigma(y)+O\left(\epsilon^{2}\right)
\end{aligned}
$$

From (3.10) and (3.11), we know that

$$
\mathcal{K}_{D}^{*}[\phi](y)+\frac{\partial H(y)}{\partial \nu(y)}=\lambda \phi(y), \quad \mathcal{K}_{D}\left[\phi_{d}\right](x, y)+\Gamma(x, y)=\lambda \phi_{d}(x, y) .
$$

Thus it follows that

$$
\begin{aligned}
\mathcal{F}\left(\partial D_{\epsilon}\right)-\mathcal{F}(\partial D) & =\epsilon \int_{\partial D} h(y)\left[\frac{\partial \phi_{d}(x, y)}{\partial T(y)}\left(\frac{\partial\left(H(y)+\mathcal{S}_{D}[\phi](y)\right)}{\partial T(y)}\right)\right. \\
& \left.+\left(\frac{\partial\left(\Gamma(x, y)+\mathcal{D}_{D}\left[\phi_{d}\right](x, y)\right)}{\partial \nu(y)}\right) \phi(y)\right] d \sigma(y)+O\left(\epsilon^{2}\right),
\end{aligned}
$$

which readily completes the proof.

In order to investigate variations in the measurement resulting from variations in the shape of the underlying object, we introduce the following definition of the shape sensitivity functional.

Definition 5. The shape sensitivity functional for the far-field measurement $\left.u^{s}\right|_{\partial \Omega}$ with respect to the shape of $\partial D$ is defined as

$$
S S F(\partial D):=\lim _{\epsilon \rightarrow 0} \frac{\left.u_{\epsilon}^{s}\right|_{\partial \Omega}-\left.u^{s}\right|_{\partial \Omega}}{\epsilon} .
$$

Remark 6. From Definition 5, it is easy to see that the shape sensitivity function is actually the shape derivative of the forward operator $\mathcal{F}(\partial D)$ (cf., [1]). Furthermore, by using Theorem 4, the shape sensitivity function can be rewritten as

$$
S S F(\partial D)=\langle h, P\rangle_{L^{2}(\partial D)}
$$

where $P$ is defined in (3.12). 


\subsection{Shape sensitivity analysis and plasmon resonance}

In this subsection, we shall derive the spectral representation of the shape sensitivity functional in this subsection. It indicates that, when the plasmon resonance occurs, the shape sensitivity functional will be amplified and exhibits a large peak. Hence, the plasmon resonance can be used to significantly increase the sensitivity of the far-field measurement with respect to the shape of the underlying domain. For simplicity, in the subsequent spectral analysis, we always exclude the essential spectrum 0 from the spectrum set of NP operator and assume the eigenvalues is simple.

First, we derive the asymptotic formulas for the eigenvalues and eigenfunctions of the NP operator with respect to the asymptotic parameter $\epsilon$.

Lemma 7. Suppose that $\left\{\lambda_{j, \epsilon}, \varphi_{j, \epsilon}\right\}, j=0,1,2, \ldots$, are the perturbed eigenvalues and eigenfunctions of $\mathcal{K}_{D_{\epsilon}}^{*}$. Then we have as $\epsilon \rightarrow 0$ that

$$
\lambda_{j, \epsilon}=\lambda_{j}+\epsilon \lambda_{j, \epsilon}^{(1)}+O\left(\epsilon^{2}\right), \quad \varphi_{j, \epsilon}=\varphi_{j}+\epsilon \varphi_{j, \epsilon}^{(1)}+O\left(\epsilon^{2}\right),
$$

where

$$
\lambda_{j, \epsilon}^{(1)}=\left\langle\mathcal{K}_{D}^{(1)} \varphi_{j}, \varphi_{j}\right\rangle_{\mathcal{H}^{*}(\partial D)}, \quad \varphi_{j, \epsilon}^{(1)}=\sum_{j \neq l} \frac{\left\langle\mathcal{K}_{D}^{(1)} \varphi_{j}, \varphi_{l}\right\rangle_{\mathcal{H}^{*}(\partial D)}}{\lambda_{j}-\lambda_{l}} \varphi_{l},
$$

and $\mathcal{K}_{D}^{(1)}$ is defined by (3.5) or (3.6).

Proof. Since $\mathcal{K}_{D_{\epsilon}}^{*}\left[\varphi_{j, \epsilon}\right]=\lambda_{j, \epsilon} \varphi_{j, \epsilon}$, and by Lemma 3.1, we can obtain the following result

$$
\left(\mathcal{K}_{D}^{*}+\epsilon \mathcal{K}_{D}^{(1)}+O\left(\epsilon^{2}\right)\right)\left(\varphi_{j}+\epsilon \varphi_{j, \epsilon}^{(1)}+O\left(\epsilon^{2}\right)\right)=\left(\lambda_{j}+\epsilon \lambda_{j, \epsilon}^{(1)}+O\left(\epsilon^{2}\right)\right)\left(\varphi_{j}+\epsilon \varphi_{j, \epsilon}^{(1)}+O\left(\epsilon^{2}\right)\right),
$$

which implies that

$$
\begin{aligned}
\mathcal{K}_{D}^{*} \varphi_{j} & =\lambda_{j} \varphi_{j}, \\
\mathcal{K}_{D}^{(1)} \varphi_{j}+\mathcal{K}_{D}^{*} \varphi_{j, \epsilon}^{(1)} & =\lambda_{j, \epsilon}^{(1)} \varphi_{j}+\lambda_{j} \varphi_{j, \epsilon}^{(1)} .
\end{aligned}
$$

By inner producting $\varphi_{l}$ in both sides of (3.14), we further have

$$
\left\langle\mathcal{K}_{D}^{(1)} \varphi_{j}, \varphi_{l}\right\rangle_{\mathcal{H}^{*}(\partial D)}+\left\langle\mathcal{K}_{D}^{*} \varphi_{j, \epsilon}^{(1)}, \varphi_{l}\right\rangle_{\mathcal{H}^{*}(\partial D)}=\lambda_{j, \epsilon}^{(1)}\left\langle\varphi_{j}, \varphi_{l}\right\rangle_{\mathcal{H}^{*}(\partial D)}+\lambda_{j}\left\langle\varphi_{j, \epsilon}^{(1)}, \varphi_{l}\right\rangle_{\mathcal{H}^{*}(\partial D)}
$$

Since the operator $\mathcal{K}_{D}^{*}$ is self-adjoint, we have

$$
\left\langle\mathcal{K}_{D}^{*} \varphi_{j, \epsilon}^{(1)}, \varphi_{l}\right\rangle_{\mathcal{H}^{*}(\partial D)}=\left\langle\varphi_{j, \epsilon}^{(1)}, \mathcal{K}_{D}^{*} \varphi_{l}\right\rangle_{\mathcal{H}^{*}(\partial D)}=\lambda_{l}\left\langle\varphi_{j, \epsilon}^{(1)}, \varphi_{l}\right\rangle_{\mathcal{H}^{*}(\partial D)}
$$

and thus

From (3.14), we have

$$
\lambda_{j, \epsilon}^{(1)}=\left\langle\mathcal{K}_{D}^{(1)} \varphi_{j}, \varphi_{j}\right\rangle_{\mathcal{H}^{*}(\partial D)} .
$$

$$
\left(\lambda_{j} I-\mathcal{K}_{D}^{*}\right) \varphi_{j, \epsilon}^{(1)}=\mathcal{K}_{D}^{(1)} \varphi_{j}-\lambda_{j, \epsilon}^{(1)} \varphi_{j} .
$$

Then it is straightforward to see that

$$
\varphi_{j, \epsilon}^{(1)}=\sum_{j \neq l} \frac{\left\langle\mathcal{K}_{D}^{(1)} \varphi_{j}, \varphi_{l}\right\rangle_{\mathcal{H}^{*}(\partial D)}}{\lambda_{j}-\lambda_{l}} \varphi_{l}
$$

The proof is complete. 
Let $\operatorname{dist}\left(\lambda, \sigma\left(\mathcal{K}_{D}^{*}\right)\right)$ be the distance of $\lambda$ to the spectrum set $\sigma\left(\mathcal{K}_{D}^{*}\right)$. Next, we are ready to state our main result in the following theorem.

Theorem 8. As $\epsilon \rightarrow 0$, the perturbation $\left.u_{\epsilon}^{s}\right|_{\partial \Omega}-\left.u\right|_{\partial \Omega}$ has the following spectral expansion:

$$
u_{\epsilon}^{s}(x)-u^{s}(x)=\epsilon \mathcal{T}(x)+O\left(\frac{\epsilon^{2}}{\operatorname{dist}\left(\lambda, \sigma\left(\mathcal{K}_{D}^{*}\right)\right)^{2}}\right), \quad x \in \partial \Omega,
$$

with

$$
\begin{aligned}
\mathcal{T}(x)= & \sum_{j=1}^{\infty} \frac{\left\langle\frac{\partial H}{\partial \nu}, \varphi_{j, \epsilon}^{(1)}\right\rangle+\left\langle G^{(1)}, \varphi_{j}\right\rangle \mathcal{S}_{D}\left[\varphi_{j}\right](x)+\left\langle\frac{\partial H}{\partial \nu}, \varphi_{j}\right\rangle \mathcal{Z}(x)}{\lambda-\lambda_{j}} \\
& +\sum_{j=1}^{\infty} \frac{\lambda_{j, \epsilon}^{(1)}\left\langle\frac{\partial H}{\partial \nu}, \varphi_{j}\right\rangle \mathcal{S}_{D}\left[\varphi_{j}\right](x)}{\left(\lambda-\lambda_{j}\right)^{2}}
\end{aligned}
$$

where $G^{(1)}$ is defined by (3.9), $\langle\cdot, \cdot\rangle$ signifies $\langle\cdot, \cdot\rangle_{\mathcal{H}^{*}(\partial D)}$, and

$$
\mathcal{Z}(x)=\int_{\partial D}\left(\frac{\partial \Gamma(x, y)}{\partial \nu(y)} h(y) \varphi_{j}(y)+\Gamma(x, y) \varphi_{j, \epsilon}^{(1)}(y)-\tau(y) h(y) \Gamma(x, y) \varphi_{j}(y)\right) d \sigma(y) .
$$

Proof. According to $(2.1), \mathcal{F}(\partial D)$ can be decomposed as follow

$$
\begin{aligned}
\mathcal{F}(\partial D) & =\int_{\partial D} \Gamma(x, y)\left(\lambda I-\mathcal{K}_{D}^{*}\right)^{-1}\left[\frac{\partial H}{\partial \nu}\right](y) d \sigma(y) \\
& =\sum_{j=1}^{\infty} \frac{\left\langle\frac{\partial H}{\partial \nu}, \varphi_{j}\right\rangle}{\lambda-\lambda_{j}} \int_{\partial D} \Gamma(x, y) \varphi_{j}(y) d \sigma(y) \\
& =\sum_{j=1}^{\infty} \frac{\left\langle\frac{\partial H}{\partial \nu}, \varphi_{j}\right\rangle \mathcal{S}_{D}\left[\varphi_{j}\right](x)}{\lambda-\lambda_{j}}
\end{aligned}
$$

In a similar manner, $\mathcal{F}\left(\partial D_{\epsilon}\right)$ can be decomposed as

$$
\begin{aligned}
\mathcal{F}\left(\partial D_{\epsilon}\right) & =\int_{\partial D_{\epsilon}} \Gamma(x, \tilde{y})\left(\lambda I-\mathcal{K}_{D_{\epsilon}}^{*}\right)^{-1}\left[\frac{\partial H}{\partial \tilde{\nu}}\right](\tilde{y}) d \tilde{\sigma}(\tilde{y}) \\
& =\sum_{j=1}^{\infty} \frac{\left\langle\frac{\partial H}{\partial \tilde{\nu}}, \varphi_{j, \epsilon}\right\rangle \mathcal{S}_{D_{\epsilon}}\left[\varphi_{j, \epsilon}\right](x)}{\lambda-\lambda_{j, \epsilon}}
\end{aligned}
$$

Furthermore, using the perturbation of eigenvalues and eigenfunctions in Lemma 7, we can show that

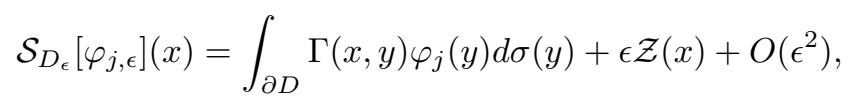

where

$$
\mathcal{Z}(x)=\int_{\partial D}\left(\frac{\partial \Gamma(x, y)}{\partial \nu(y)} h(y) \varphi_{j}(y)+\Gamma(x, y) \varphi_{j, \epsilon}^{(1)}(y)-\tau(y) h(y) \Gamma(x, y) \varphi_{j}(y)\right) d \sigma(y) .
$$

From (3.8), (3.16) and Lemma 7, we deduce that

$$
\mathcal{F}\left(\partial D_{\epsilon}\right)=\sum_{j=1}^{\infty} \frac{\left\langle\frac{\partial H}{\partial \tilde{\nu}}, \varphi_{j, \epsilon}\right\rangle \mathcal{S}_{D_{\epsilon}}\left[\varphi_{j, \epsilon}\right](x)}{\lambda-\lambda_{j, \epsilon}}
$$




$$
\begin{aligned}
= & \sum_{j=1}^{\infty} \frac{\left\langle\frac{\partial H}{\partial \nu}+\epsilon G^{(1)}+O\left(\epsilon^{2}\right), \varphi_{j}+\epsilon \varphi_{j, \epsilon}^{(1)}+O\left(\epsilon^{2}\right)\right\rangle\left(\mathcal{S}_{D}\left[\varphi_{j}\right](x)+\epsilon \mathcal{Z}(x)+O\left(\epsilon^{2}\right)\right)}{\lambda-\lambda_{j}-\epsilon \lambda_{j, \epsilon}^{(1)}+O\left(\frac{\epsilon^{2}}{\lambda-\lambda_{j}}\right)} \\
= & \sum_{j=1}^{\infty} \frac{\left\langle\frac{\partial H}{\partial \nu}, \varphi_{j}\right\rangle \mathcal{S}_{D}\left[\varphi_{j}\right](x)+\epsilon\left(\left\langle\frac{\partial H}{\partial \nu}, \varphi_{j, \epsilon}^{(1)}\right\rangle \mathcal{S}_{D}\left[\varphi_{j}\right](x)+\left\langle G^{(1)}, \varphi_{j}\right\rangle \mathcal{S}_{D}\left[\varphi_{j}\right]+\left\langle\frac{\partial H}{\partial \nu}, \varphi_{j}\right\rangle \mathcal{Z}(x)\right)+O\left(\epsilon^{2}\right)}{\left(\lambda-\lambda_{j}\right)\left(1-\frac{\epsilon \lambda_{j, \epsilon}^{(1)}}{\lambda-\lambda_{j}}+O\left(\frac{\epsilon^{2}}{\lambda-\lambda_{j}}\right)\right)} \\
= & \left(\sum_{j=1}^{\infty}\left\langle\frac{\partial H}{\partial \nu}, \varphi_{j}\right\rangle \mathcal{S}_{D}\left[\varphi_{j}\right](x)+\epsilon\left(\left\langle\frac{\partial H}{\partial \nu}, \varphi_{j, \epsilon}^{(1)}\right\rangle \mathcal{S}_{D}\left[\varphi_{j}\right](x)+\left\langle G^{(1)}, \varphi_{j}\right\rangle \mathcal{S}_{D}\left[\varphi_{j}\right](x)\right.\right. \\
& \left.\left.+\left\langle\frac{\partial H}{\partial \nu}, \varphi_{j}\right\rangle \mathcal{Z}(x)\right)+O\left(\epsilon^{2}\right)\right) \cdot\left(\sum_{k=0}^{\infty} \frac{1}{\left(\lambda-\lambda_{j}\right)}\left(\frac{\epsilon \lambda_{j, \epsilon}^{(1)}}{\lambda-\lambda_{j}}+O\left(\frac{\epsilon^{2}}{\lambda-\lambda_{j}}\right)\right)^{k}\right) \\
= & \sum_{j=1}^{\infty} \frac{\left\langle\frac{\partial H}{\partial \nu}, \varphi_{j}\right\rangle \mathcal{S}_{D}\left[\varphi_{j}\right](x)}{\lambda-\lambda_{j}}+\epsilon\left(\frac{\left\langle\frac{\partial H}{\partial \nu}, \varphi_{j, \epsilon}^{(1)}\right\rangle \mathcal{S}_{D}\left[\varphi_{j}\right](x)+\left\langle G^{(1)}, \varphi_{j}\right\rangle \mathcal{S}_{D}\left[\varphi_{j}\right](x)+\left\langle\frac{\partial H}{\partial \nu}, \varphi_{j}\right\rangle \mathcal{Z}(x)}{\lambda-\lambda_{j}}\right. \\
& \left.+\frac{\left\langle\frac{\partial H}{\partial \nu}, \varphi_{j}\right\rangle \mathcal{S}_{D}\left[\varphi_{j}\right](x) \lambda_{j, \epsilon}^{(1)}}{\left(\lambda-\lambda_{j}\right)^{2}}\right)+O\left(\frac{\epsilon^{2}}{\operatorname{dist}\left(\lambda, \sigma\left(\mathcal{K}_{D}^{*}\right)\right)^{2}}\right) .
\end{aligned}
$$

Therefore it follows that

$$
\begin{aligned}
\mathcal{F}\left(\partial D_{\epsilon}\right)-\mathcal{F}(\partial D) & =\epsilon \sum_{j=1}^{\infty} \frac{\left\langle\frac{\partial H}{\partial \nu}, \varphi_{j, \epsilon}^{(1)}\right\rangle+\left\langle G^{(1)}, \varphi_{j}\right\rangle \mathcal{S}_{D}\left[\varphi_{j}\right](x)+\left\langle\frac{\partial H}{\partial \nu}, \varphi_{j}\right\rangle \mathcal{Z}(x)}{\lambda-\lambda_{j}} \\
& +\epsilon \sum_{j=1}^{\infty} \frac{\left\langle\frac{\partial H}{\partial \nu}, \varphi_{j}\right\rangle \mathcal{S}_{D}\left[\varphi_{j}\right](x) \lambda_{j, \epsilon}^{(1)}}{\left(\lambda-\lambda_{j}\right)^{2}}+O\left(\frac{\epsilon^{2}}{\operatorname{dist}\left(\lambda, \sigma\left(\mathcal{K}_{D}^{*}\right)\right)^{2}}\right) .
\end{aligned}
$$

The proof is complete.

Remark 9. From (3.15), one readily sees that the $j$-th mode in the expansion formula contains both $\mathcal{O}\left(\frac{1}{\lambda-\lambda_{j}}\right)$ and $\mathcal{O}\left(\frac{1}{\left(\lambda-\lambda_{j}\right)^{2}}\right)$. Thus, for the sufficiently small loss $\left(\mathfrak{I m}\left(\varepsilon_{D}\right) \rightarrow 0\right)$, the $j$-th mode will exhibit a large peak if the plasmon resonance condition (2.6) is fulfilled.

From Theorem 8 , we can straightforwardly obtain the spectral representation formula of the shape sensitivity function as follows.

Corollary 10. If $\epsilon=o\left(\operatorname{dist}\left(\lambda, \sigma\left(\mathcal{K}_{D}^{*}\right)\right)^{2}\right)$, (as $\left.\operatorname{dist}\left(\lambda, \sigma\left(\mathcal{K}_{D}^{*}\right)\right) \rightarrow 0\right)$, the shape sensitivity function can be represented as

$$
S S F(\partial D)=\mathcal{T}(x)
$$

where $\mathcal{T}(x)$ is defined by (3.15).

Remark 11. Similar to Remark 9, one sees that when $\lambda=\frac{\varepsilon_{D}+\varepsilon_{m}}{2\left(\varepsilon_{D}-\varepsilon_{m}\right)}$ is very close to an eigenvalue $\lambda_{j}$ of the NP operator, i.e., the plasmon resonance occurs, the shape sensitivity function $S S F(\partial D)$ will be amplified dramatically for the $j$-th mode. Hence, the plasmon resonance can improve the sensitivity of the shape construction. Moreover, from the Drude's model (2.4), we can compute the plasmon resonance frequency for improving the sensitivity.

Remark 12. From a numerical point of view, the discrete version of the shape sensitivity functional is the shape sensitivity matrix. The sensitivity of the shape reconstruction can be analysed by the singular value decomposition of the sensitivity matrix. In Section 5, the numerical results shall show that when the plasmon resonance occurs, the singular values of the sensitivity matrix increase dramatically. That is the plasmon resonance technology can enhance the stability of the Gauss-Newton iteration algorithm that we use therein (see Sect. 4). 


\section{TikHONOV REGULARIZATION AND LAPLACE APPROXIMATION}

In this section, we discuss the numerical issues for the shape reconstruction. First, a numerical implementation requires a parametrization of the boundary $\partial D$. Here we assume that $\partial D$ is starlike boundary curve with respect to the origin, i.e., there exists $q \in C^{2}[0,2 \pi]$ such that

$$
\partial D=\left\{\boldsymbol{q}(t)=q(t)\left(\begin{array}{c}
\cos t \\
\sin t
\end{array}\right), t \in[0,2 \pi]\right\} .
$$

The admissible set $X=\left\{q \in C^{2}([0,2 \pi]): q>0\right\}$, and the forward operator maps $X$ into $H^{\frac{1}{2}}(\partial \Omega)$. Without change of notation we write $\mathcal{F}(q)=\left.u^{s}(x)\right|_{\partial \Omega}$.

In order to overcome the ill-posedness of the inverse problem, we apply the Tikhonov regularization method to tackle it. The corresponding variational functional is given as follows,

$$
J[q]:=\frac{1}{2}\left\|\mathcal{F}(q)-u^{s, \delta}\right\|_{2}^{2}+\frac{\mu}{2}\|q\|_{L^{2}[0,2 \pi]}^{2},
$$

where, $u^{s, \delta}=\left(u_{1}^{s, \delta}, u_{2}^{s, \delta}, \ldots, u_{n}^{s, \delta}\right)$ signfies the discrete measurement data on $\partial \Omega$. Here, $\|\cdot\|_{2}$ denotes the 2-norm in $\mathbb{R}^{n}$, and $\mu$ is the regularization parameter. Moreover, the measurement data $u^{s, \delta}$ and the exact data $u^{s}$ satisfies $\left\|u^{s, \delta}-u^{s}\right\|_{2} \leq \delta$.

There are many iterative algorithms to solve the variational problem (4.1). In this paper, we apply the Levenberg-Marquardt method [26,32] to find the minimize of $J[q]$, which is essentially a variant of the GaussNewton iteration. Assuming that $q^{*}$ is an approximation of $q$, then the nonlinear mapping $\mathcal{F}$ in (4.1) can be replaced approximatively by its linearization around $q^{*}$, i.e.,

$$
\mathcal{F}(q) \approx \mathcal{F}\left(q^{*}\right)+\mathcal{F}^{\prime}\left(q^{*}\right)\left(q-q^{*}\right)
$$

The nonlinear inverse problem $\mathcal{F}(q)=u^{s, \delta}$ can then be converted to a linear inverse problem

$$
\mathcal{F}^{\prime}\left(q^{*}\right)\left(q-q^{*}\right)=u^{s, \delta}-\mathcal{F}\left(q^{*}\right) .
$$

Thus, minimizing (4.1) can easily be seen to minimizing

$$
J[q]:=\frac{1}{2}\left\|\mathcal{F}^{\prime}\left(q^{*}\right) \delta q-\left(u^{s, \delta}-\mathcal{F}\left(q^{*}\right)\right)\right\|_{2}^{2}+\frac{\mu}{2}\|\delta q\|_{L^{2}[0,2 \pi]}^{2},
$$

where, $\delta q=q-q^{*}$. We formulate the Levenberg-Marquardt iteration as Algorithm 1 .

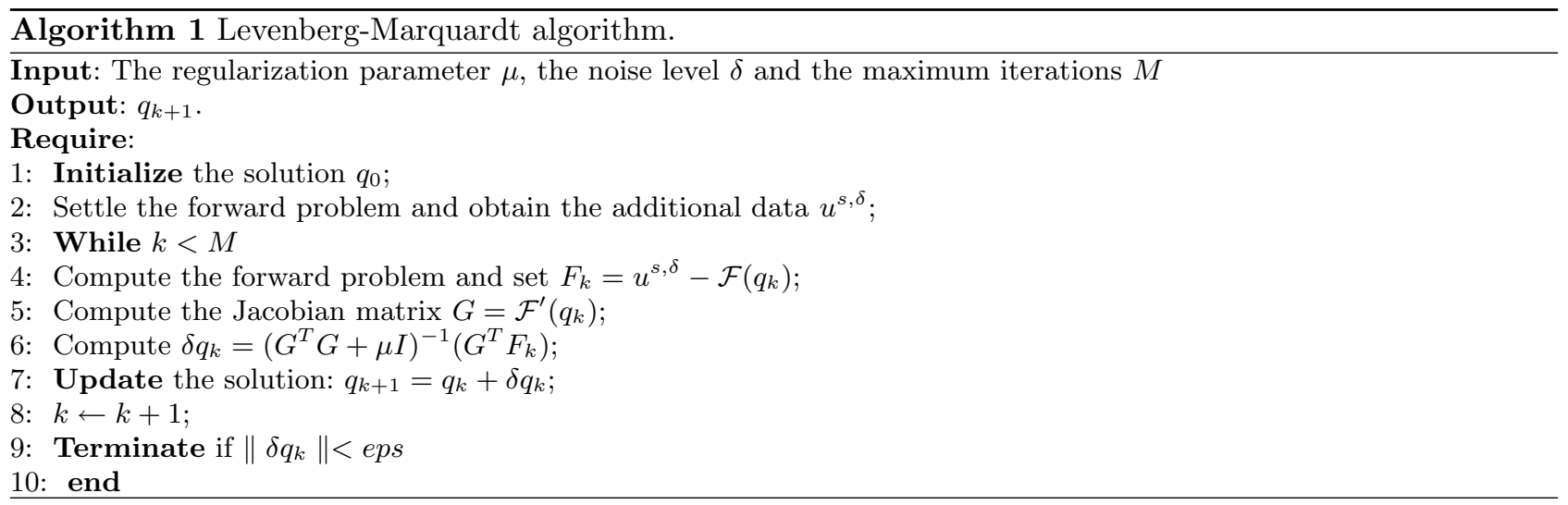


We also decipher the inverse problem from a Bayesian perspective so that we can capture more statistical information about the solution. From the classical Bayesian theory, and noticing that the observation error $\xi$ is assumed to be an independent and identically distributed Gauss random vector with mean zero and the covariance matrix $B=\delta^{2} I$ (here $I$ is the unit matrix), we can write the minimization functional as

$$
\begin{aligned}
J[q] & \propto \frac{1}{2 \delta^{2}}\left\|\mathcal{F}(q)-u^{\delta}\right\|_{2}^{2}+\frac{\mu}{2 \delta^{2}}\|q\|_{L^{2}[0,2 \pi]}^{2} \\
& =\frac{1}{2}\left\|\mathcal{F}(q)-u^{\delta}\right\|_{B}^{2}+\frac{\mu}{2 \delta^{2}}\|q\|_{L^{2}[0,2 \pi]}^{2} \\
& =: J_{B}(q),
\end{aligned}
$$

where $\|\cdot\|_{B}$ is a covariance weighted norm given by $\|\cdot\|_{B}=\left\|\delta^{-1} I \cdot\right\|_{2}$, and the minimizer of $J_{B}$ defines the maximum a posteriori estimator

$$
q_{\mathrm{MAP}}=\arg \min _{q} J_{B}(q)
$$

The Laplace approximation replaces the complicated posterior with a normal distribution located at the maximum a posteriori value $q_{\mathrm{MAP}}$. Its essence is a linearization around the MAP point $q_{\mathrm{MAP}}(c f .,[52])$. It consists of approximating the posterior measure (or distribution) by $\omega \approx N\left(q_{\mathrm{MAP}}, C_{\mathrm{MAP}}\right)$, where

$$
C_{\mathrm{MAP}}=\left(J_{B}^{\prime \prime}\right)^{-1}=\left(\frac{\mu}{\delta^{2}} I+\frac{1}{\delta^{2}} G^{T} G\right)^{-1},
$$

and $G$ is the Jacobian matrix of the forward operator $\mathcal{F}$ at the point $q$. Notice that the covariance formula (4.3) only uses the first order derivatives of $\mathcal{F}$. The implementation of the Laplace approximation is presented in the following algorithm [35].

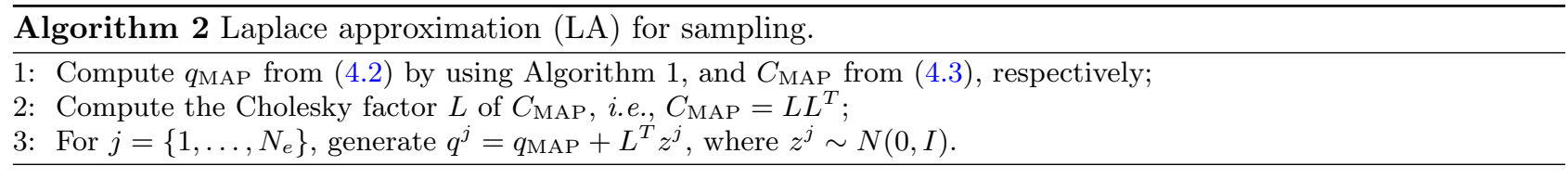

In this algorithm, samples generated by algorithm 2 are drawn from $N\left(q_{\mathrm{MAP}}, C_{\mathrm{MAP}}\right)$, and so the ensemble of $N_{e}$ realizations $\left\{q_{j}\right\}_{j=1}^{N_{e}}$ provides an approximation to $N\left(q_{\mathrm{MAP}}, C_{\mathrm{MAP}}\right)$ and hence the posterior. Finally, we use the mean of the sample $\bar{q}=\frac{1}{N_{e}} \sum_{j=1}^{N_{e}} q_{j}$ as an approximation of $q_{\mathrm{MAP}}$, where the convergence of $\bar{q}$ follows from the strong law of large numbers. From the classical Gaussian statistic theory, we find that $\bar{q}$ is consistent and the best unbiased estimate of $q_{\mathrm{MAP}}$.

\section{NUMERICAL RESUlTS AND DISCUSSIONS}

In this section, we present several numerical examples to illustrate the salient and promising features of the proposed reconstruction scheme.

In all of our numerical examples, the measurement boundary curve $\partial \Omega$ is given by the circle of radius 3 and centred at the origin, that is $\partial \Omega=\{3(\cos t, \sin t), 0 \leq t \leq 2 \pi\}$. Set the incident field $H(x)=x_{1}$. We solve the direct problem through the Nyström method [37], which is discretized with $n=80$ grid points. Furthermore, in an effort to avoid committing an inverse crime, the number of collocation points for obtaining the synthetic data was chosen to be different from the number of collocation points within the inverse solver. In addition, we approximate the radial function $q(t)$ for unknown interior boundary curve $\partial D$ by the trigonometric series

$$
q(t) \approx \sum_{k=0}^{m} a_{k} \cos k t+\sum_{k=1}^{m} b_{k} \sin k t, \quad 0 \leq t \leq 2 \pi,
$$


where $m \in \mathbb{N}$ and the vector $q=\left(a_{0}, \ldots, a_{m}, b_{1}, \ldots, b_{m}\right) \in \mathbb{R}^{2 m+1}$.

In the iterative process, we choose a circle as the initial guess, which contains the inclusion $D$. A finite difference method is used to calculate the Jacobian matrix $G$, and the maximum number of iteration steps is 100. The number of samples $N_{e}$ is 10000 , and we use the following stopping rule

$$
E_{k}=\left\|q_{k}-q_{k-1}\right\|_{L^{2}} \leq 10^{-5} .
$$

The noisy measured data are generated by

$$
u^{s, \delta}=u^{s}(x)+\delta \xi, \quad x \in \partial \Omega,
$$

where $u^{s}(x)$ is the exact data, $\delta$ indicates the noise level, and $\xi$ is the Gaussian random vector with a zero mean and unit standard deviation.

The accuracy of the approximate solution $\tilde{q}_{N_{e}}$ by the LA algorithm is characterised by comparing to the exact solution $q(x)$ via the relative error

$$
e_{\gamma}=\frac{\left\|\tilde{q}_{N_{e}}-q(x)\right\|_{L^{2}(\partial D)}}{\|q(x)\|_{L^{2}(\partial D)}}
$$

In particular, we specifically take the permittivity $\varepsilon_{m}=24.8 \mathrm{~F} / \mathrm{m}$ (such as alcohol) in the first example, and in all of the examples we set $\varepsilon_{m}=\varepsilon_{0}$. Moreover, except for Example 13, the spectrum of $\mathcal{K}_{D}^{*}$ needs to calculated numerically, from which one can then compute the corresponding plasmon resonance frequency. We use the Nyström method for spectral calculations. However, we would like to emphasise that in practice, the plasmon resonance frequency can be measured by special apparatus, when one can observe significant enhancements in the electric field due to the resonance.

Example 13. In this example, we consider the reconstruction of a circle object with

$$
q(t)=0.5, \quad 0 \leq t \leq 2 \pi
$$

In the iteration algorithm, we choose a circle of radius 0.6 as the initial guess.

First, we investigate the influence of different $\lambda$ on the reconstruction effect. The numerical results for Example 13 with $\lambda$ taking different values are shown in Figure $1 \mathrm{a}$. It is well known that the eigenvalues of $\mathcal{K}_{D}^{*}$ are $\left\{0, \frac{1}{2}\right\}$ for $D$ being a disk. If setting $\lambda_{1}=0$, from Section 2.2, we can see that the Fröhlich condition is satisfied based on the Drude's model for metal nanorod and the plasmon resonance occurs (though our theoretical analysis does not contain the essential spectrum case).

In particular, we take the resonance frequency $\omega_{1}=3.63 \times 10^{14} \mathrm{~Hz}$, and according to the experimental data of gold nanorod in [49] (the data coincide with the Drude model), we can get $\mathfrak{R e}\left(\varepsilon_{D}\left(\omega_{1}\right)\right)=-24.8$, and the imaginary part $\mathfrak{I m}\left(\varepsilon_{D}\left(\omega_{1}\right)\right)=0.797$. Furthermore, by $(2.3)$, it implies that $\lambda \approx 0-8 \times 10^{-3} i$.

Next, we take $\omega_{2}=2.30 \times 10^{14} \mathrm{~Hz}$ and based on the experimental data in [49], we can calculate $\lambda=$ $0.244-6.3 \times 10^{-3} i$. It is obvious that when taking $\omega_{2}$, the real part of $\lambda\left(\omega_{2}\right)$ satisfies $\left|\mathfrak{R e}\left(\lambda\left(\omega_{2}\right)\right)<\frac{1}{2}\right|$, which is only an approximate resonance frequency. As seen in Figure 1a, a perfect reconstruction result is obtained when taking the resonance frequency $\omega_{1}$. When $\lambda=0.244-6.3 \times 10^{-3} i$, although the reconstructed result is good, it is still poor compared to the case with the resonant frequency $\omega_{1}$. The case with $\lambda=-0.98$ indicates a normal material, i.e., the permittivity $\varepsilon_{D}=8$ is positive constant, and the reconstruction effect is poorer. The plot in Figure 1b illustrates the relative error $e_{\gamma}$ versus the iteration number with different $\lambda$. It can be seen that the relative error can quickly reach the convergence and remain small when $\omega_{1}$ is the plasmon resonance frequency. When $\lambda=0.244-6.3 \times 10^{-3} i$, the relative error also converges quickly, but is larger than that at the plasmon resonance frequency. In particular, the relative error increases rapidly even up to $27 \%$, and the inversion is rather imprecise with $\lambda=-0.98$. 


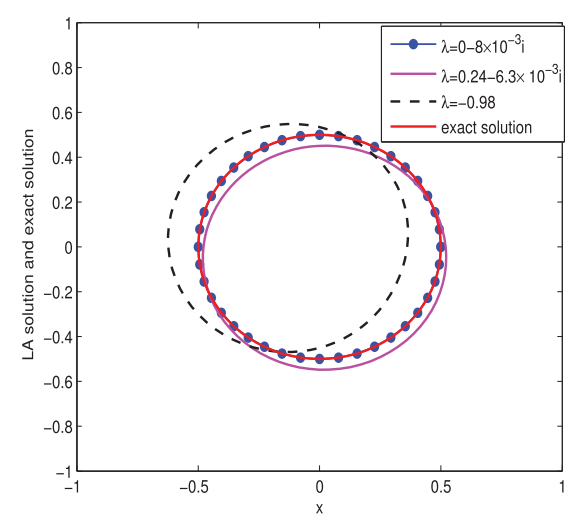

(a)

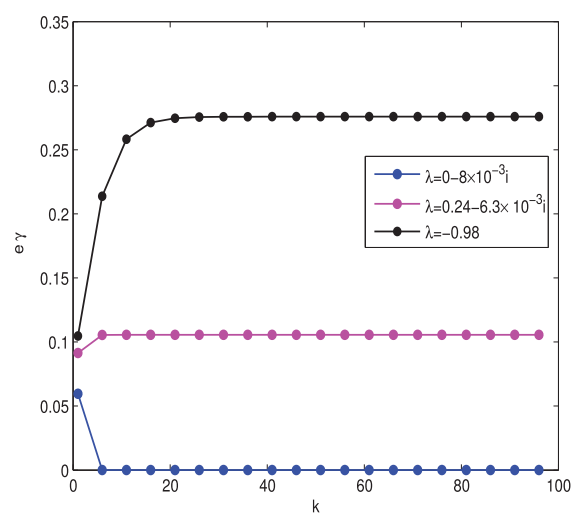

(b)

FiguRE 1. Reconstruction the shape for Example 13 with $1 \%$ noise data and the regularization parameter $\mu=0.01$. (a) Reconstructions with different $\lambda$ as well as the exact solution, (b) the relative error $e_{\gamma}$ versus the iterations step $k$, associated to different values of $\lambda$.

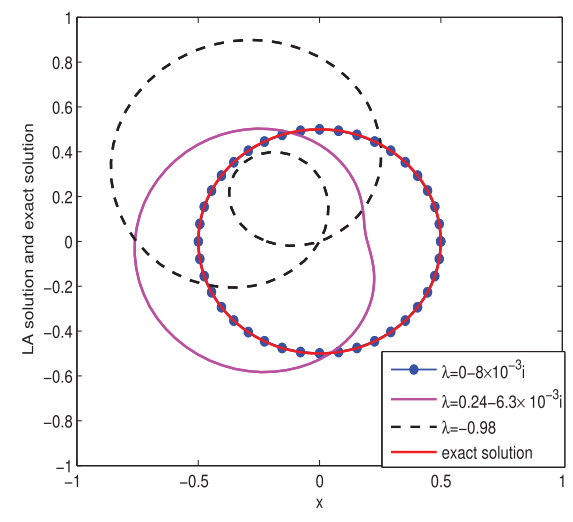

(a)

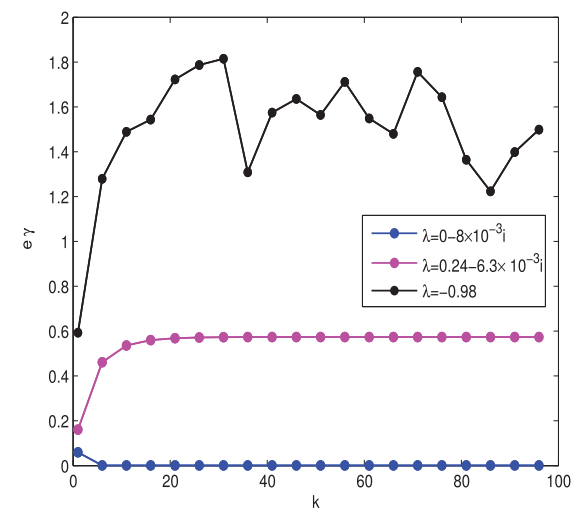

(b)

Figure 2. Reconstruction of the shape for Example 13 with $5 \%$ noise data and the regularization parameter $\mu=0.05$. (a) Reconstruction under different $\lambda$ as well as the exact solution, (b) the accuracy error $e_{\gamma}$ of the number of the iterations $k$, for different values of $\lambda$.

The numerical results for Example 13, which has $5 \%$ noise in the data, are shown in Figure 2a for different $\lambda$. Compared to the $1 \%$ noise in the data shown in Figure 1a, it is clear that as the noise level increases, the reconstruction effect becomes worse. It is worth noting that when $\omega$ is a plasmon resonance frequency, the reconstruction is very good even at larger error levels. For $\lambda=-0.98$ (general materials) the inversion result deviates from the exact solution quickly as the error level increases. We also list the relative errors for different noise levels in Table 1. 
TABLE 1. Numerical results of Example 13 for $e_{\gamma}$ associated with different $\lambda$ and noise level $\delta$.

\begin{tabular}{ccc}
\hline \hline$\lambda$ & $\delta=0.01$ & $\delta=0.05$ \\
\hline $0-8 \times 10^{-3} i$ & $7.2 \times 10^{-5}$ & $9.4 \times 10^{-4}$ \\
\hline $0.24-6.3 \times 10^{-3} i$ & 0.106 & 0.574 \\
\hline-0.98 & 0.276 & 1.546 \\
\hline
\end{tabular}

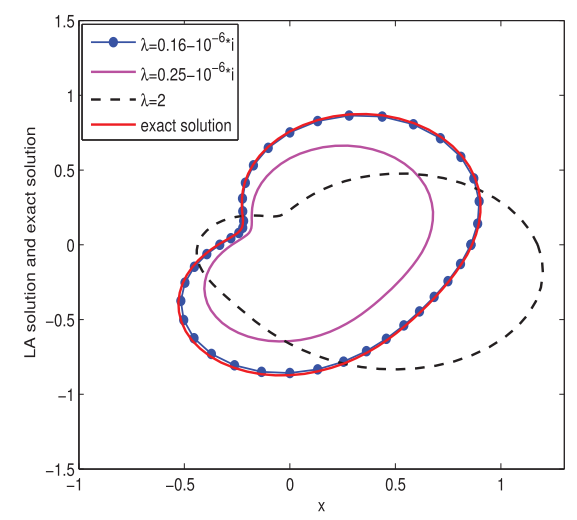

(a)

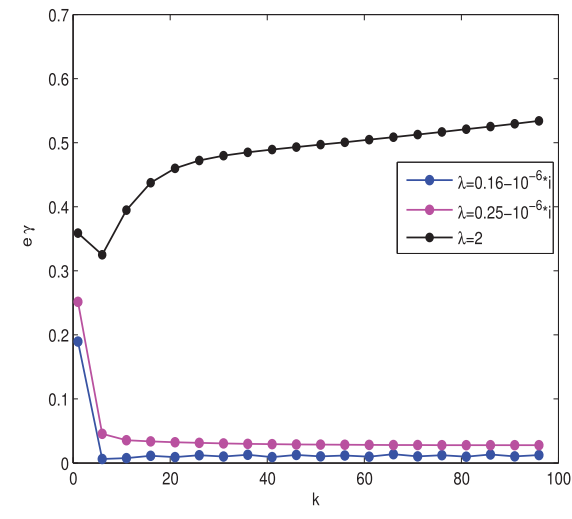

(b)

FIgURE 3. Reconstruction of the shape in Example 14 with $1 \%$ noise data and the regularization parameter $\mu=0.1$. (a) Reconstruction with different $\lambda$ as well as the exact solution, (b) the relative error $e_{\gamma}$ versus the iteration step $k$ associated with different values of $\lambda$.

TABLE 2. Numerical results of Example 14 for $e_{\gamma}$ associated with different $\lambda$ and $\delta$.

\begin{tabular}{ccc}
\hline \hline$\lambda$ & $\delta=0.01$ & $\delta=0.05$ \\
\hline $0.16-10^{-6} i$ & 0.0081 & 0.1237 \\
\hline $0.25-10^{-6} i$ & 0.0278 & 0.2810 \\
\hline 2 & 0.5304 & 0.5804 \\
\hline
\end{tabular}

Example 14. In this example, we consider the reconstruction of a bean contour with a radial function,

$$
q(t)=\frac{4 / 5+18 / 25 \cos t+3 / 25 \sin 2 t}{1+7 / 10 \cos t}, \quad 0 \leq t \leq 2 \pi
$$

We choose a circle of radius 0.72 as the initial guess.

The numerical results for Example 14 with $\lambda$ taking different values are shown in Figure 3a and the relative error $e_{\gamma}$ versus the iteration number with different $\lambda$ in Figure $3 \mathrm{~b}$. The real part of $\lambda=0.16-10^{-6} i$ is the eigenvalue of $\mathcal{K}_{D}^{*}$ in Example 14. It represents very accurate approximations of the LA solution to the exact solution, and in the iteration algorithm, the relative error quickly reaches a stable state and remains small. In addition, when $\lambda=2$, the reconstruction is bad. The results show that the relative error becomes more oscillating as the noise level $\delta$ increases; see Figure 4 and Table 2 for $e_{\gamma}$ with different $\lambda$ and $\delta$. 


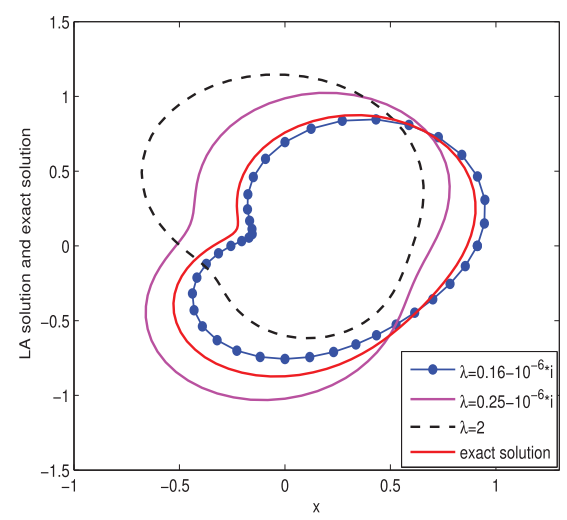

(a)

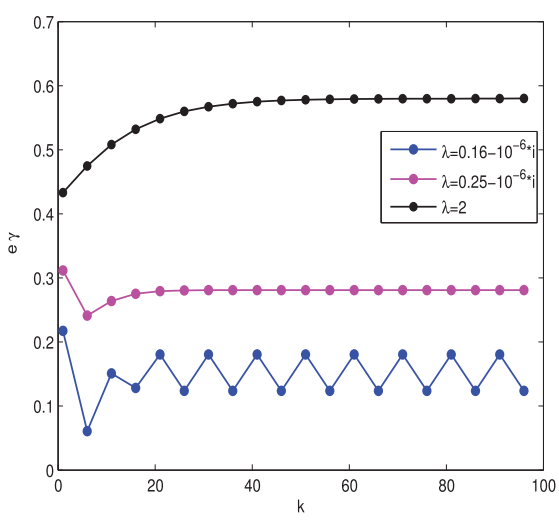

(b)

FIGURE 4. Reconstruction of the shape in Example 14 with $5 \%$ noise data and the regularization parameter $\mu=0.1$. (a) Reconstruction with different $\lambda$ as well as the exact solution, (b) the relative error $e_{\gamma}$ versus iteration steps $k$ associated with different values of $\lambda$.

Example 15. In this example, we consider that the inclusion is a peanut, and the polar radius of the peanut is parameterized by

$$
q(t)=\sqrt{\cos ^{2} t+0.26 \sin ^{2}(t+0.5)}, \quad 0 \leq t \leq 2 \pi .
$$

In the iteration, we choose a circle of radius 0.78 as the initial guess.

From [19], it is known that the singular value decomposition of the sensitivity matrix plays a key role in the uncertainty estimation. Let the singular value decomposition (SVD) of the sensitivity matrix (Jacobian matrix) $G$ of the forward operator at the true solution $q_{\text {true }}$ be denoted as

$$
G\left(q_{\text {true }}\right)=U\left[\begin{array}{c}
\Lambda \\
0
\end{array}\right] V^{T}
$$

where $U$ is an $n \times n$ orthogonal matrix, i.e., $U^{T} U=U U^{T}=I_{n}$, with $U_{1}$ containing the first $2 m+1$ columns of $U$ and $U_{2}$ containing the last $n-(2 m+1)$ columns, $U=\left[\begin{array}{ll}U_{1} & U_{2}\end{array}\right]$. The matrix $V$ is an $(2 m+1) \times(2 m+1)$ orthogonal matrix, i.e., $V^{T} V=V V^{T}=I_{p}$, and $v_{i}$ and $u_{i}$ denote the $i$ th columns of $V$ and $U$, respectively. The diagonal matrix $\Lambda=\operatorname{diag}\left(s_{1}, \ldots, s_{2 m+1}\right)$ with strictly positive decreasing singular values $s_{i}, i . e ., s_{1} \geq s_{2} \geq$ $\ldots \geq s_{2 m+1} \geq 0$. Then the estimator $q$ has the following form [19]:

$$
q=q_{\text {true }}+V \Lambda^{-1} U_{1}^{T} \tilde{\xi}=q_{\text {true }}+\sum_{i=1}^{2 m+1} \frac{1}{s_{i}} v_{i} u_{i}^{T} \tilde{\xi}
$$

From (5.1), it can be seen that the instability of the inverse problem is caused by the small singular values. In Example 15, the singular values of the sensitivity matrix are calculated at different values of $\lambda$. In Figure $5 \mathrm{~b}$, when $\lambda(\omega)=0.19-10^{-6} i$ ( $\omega$ is a plasmon resonance frequency for Example 15), we can see that all the singular values of $G$ are lager than $\lambda=0.25-10^{-6} i$ and $\lambda=2$. Consequently, the numerical solution computed by using the plasmon resonance is in an excellent agreement with the exact solution (see Fig. 5a). Hence, the plasmon resonance of the metal nanoparticles can correct the singular value of the sensitivity matrix and overcome the numerical instability. This numerical result is in agreement with our analysis in Section 3.2 that the sensitivity 


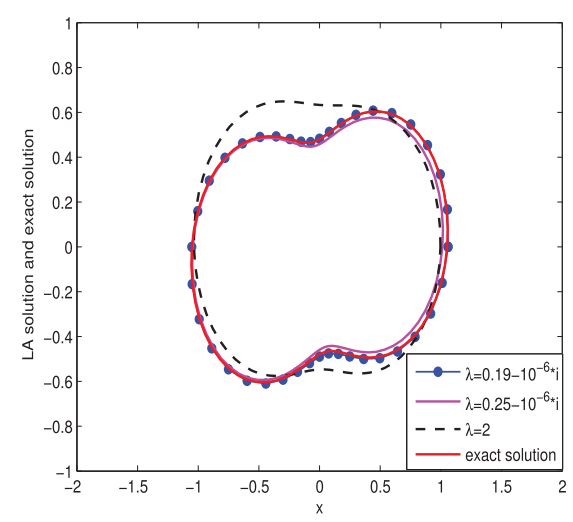

(a)

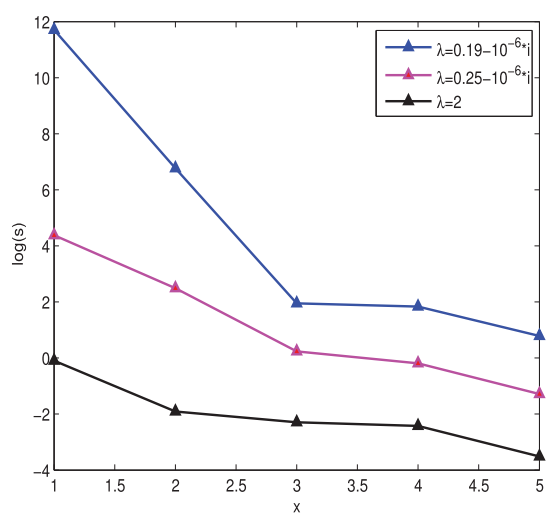

(b)

FiguRE 5. (a) Reconstruction of the shape in Example 15 with $1 \%$ noise data and different $\lambda$ as well as the exact solution, under the same regularization parameter $\mu=0.05$, (b) the distribution of the singular values associated to different values of $\lambda$.

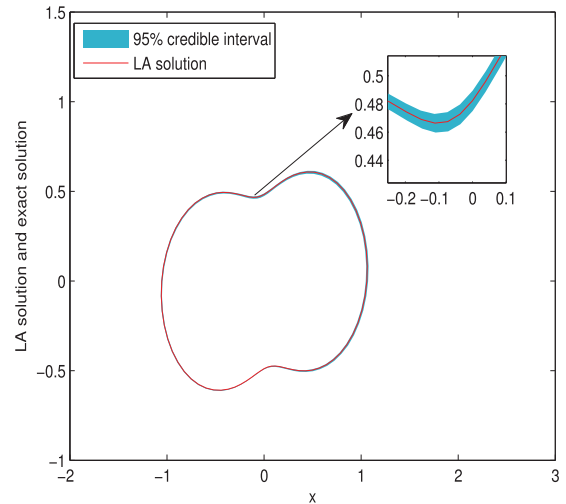

(a)

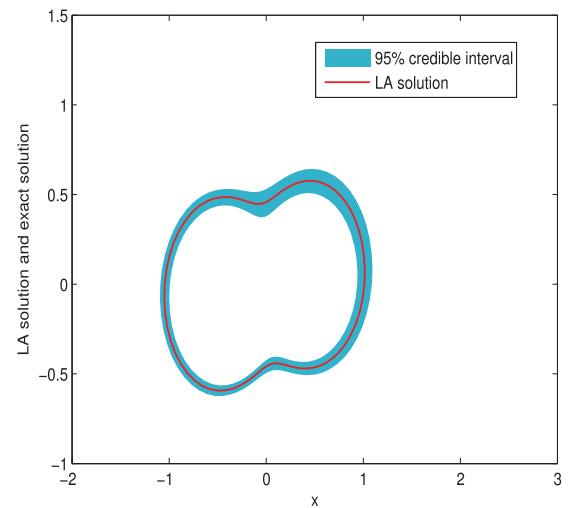

(b)

Figure 6 . The numerical results for Example 15 with $1 \%$ noise in the data and $95 \%$ confidence interval for different $\lambda$. (a) $\lambda=0.19-10^{-6} i$. (b) $\lambda=2$.

of the far-field data to the shape of the underlying domain can be enhanced at the resonant frequencies, thus reducing the ill-posedness of the inverse problem.

Next, we study the variations of the confidence intervals with $\operatorname{different} \operatorname{dist}\left(\lambda, \sigma\left(\mathcal{K}_{D}^{*}\right)\right)$. The confidence interval can quantify the uncertain information of the solution. The numerical results for Example 15 with different $\lambda$ are shown in Figure 6, where the blue region represents the corresponding 95\% confidence region. The comparison with $\lambda=2$ indicates that the confidence region shrinks at $\lambda=0.19-10^{-6} i$ (the real part of $\lambda=0.19-10^{-6} i$ is the eigenvalue of $\mathcal{K}_{D}^{*}$ in Example 15). It can be clearly obtained that as $\operatorname{dist}\left(\lambda, \sigma\left(\mathcal{K}_{D}^{*}\right)\right)$ shrinks, the accuracy of the inversion can be improved and the sensitivity to the random error can be reduced.

Example 16. In this example, we consider that the inclusion is pear, and the polar radius of the pear is parameterized by,

$$
q(t)=18 / 25+3 / 20 \cos (3 t), \quad 0 \leq t \leq 2 \pi .
$$




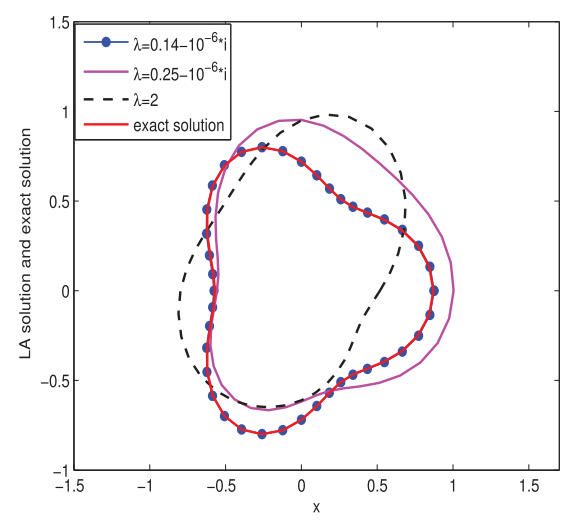

(a)

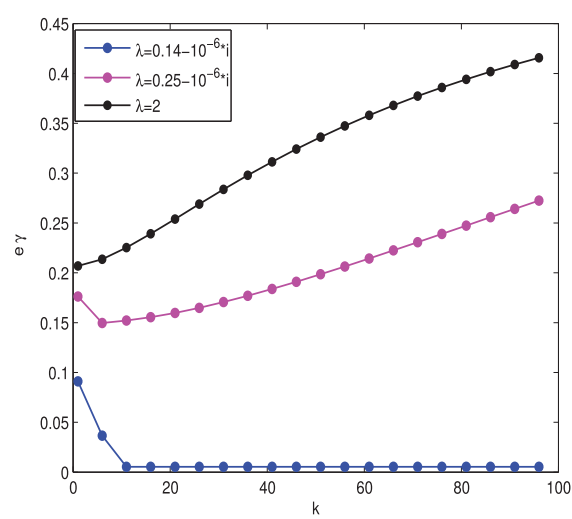

(b)

Figure 7. Reconstruction the shape for Example 16 with $1 \%$ noise data and regularization parameter $\mu=0.5$. (a) Reconstruction with different $\lambda$ and exact solution, (b) the relative error $e_{\gamma}$ versus iterations steps $k$, for various values of $\lambda$.

TABLE 3. Numerical results of Example 16 for $e_{\gamma}$ with various $\lambda$ and noise level $\delta$.

\begin{tabular}{ccc}
\hline \hline$\lambda$ & $\delta=0.01$ & $\delta=0.05$ \\
\hline $0.14-10^{-6} i$ & 0.0054 & 0.0625 \\
\hline $0.25-10^{-6} i$ & 0.279 & 0.4850 \\
\hline 2 & 0.4206 & 1.009 \\
\hline
\end{tabular}

In the iteration, we choose a circle of radius 0.73 as the initial guess.

In Example 16, we consider reconstructing a more challenging pear-shaped inclusion and we can actually reach similar conclusions to previous three Examples. At the same noise level, $\tilde{q}_{N_{e}}$ coincides well with the exact solution when $\lambda=0.14-10^{-6} i$ (minimum distance of $\operatorname{dist}\left(\lambda, \sigma\left(\mathcal{K}_{D}^{*}\right)\right.$ ), and a steady, fast convergence of the relative error $e_{\gamma}$ in the iteration algorithm is shown in Figure 7. Moreover, the relative error becomes more oscillating as the noise level $\delta$ increases; see Table 3 for $e_{\gamma}$ associated with different $\lambda$ and $\delta$.

The choice of the regularization parameters is very important in the algorithmic calculation. In Example 16, we fixed the regularization parameter $\mu=0.5$ associated to different values of $\lambda$. In order to eliminate the influence of improper regularization parameters on the inversion results, the regularization parameters can be considered as a random variable with uniform distribution, i.e., $\mu \sim U(0,1)$. In fact, there are many other selection strategies of regularization parameters, such as Morozovs discrepancy principle, The L-curve method and so on (see [54]). But we only concern the comparative effectiveness of the plasmon resonance case and the non-resonance case, and then above simple regularization parameters selection is used. The more advanced selection strategies will be considered in our future works. In Figure 8, when $\lambda=2$, we obtain four groups of random numbers from uniform distribution as the regularization parameters, while the corresponding reconstruction results remain disappointing. Table 4 lists 10 randomly generated regularization parameters with the relative error also exceeding $50 \%$. 


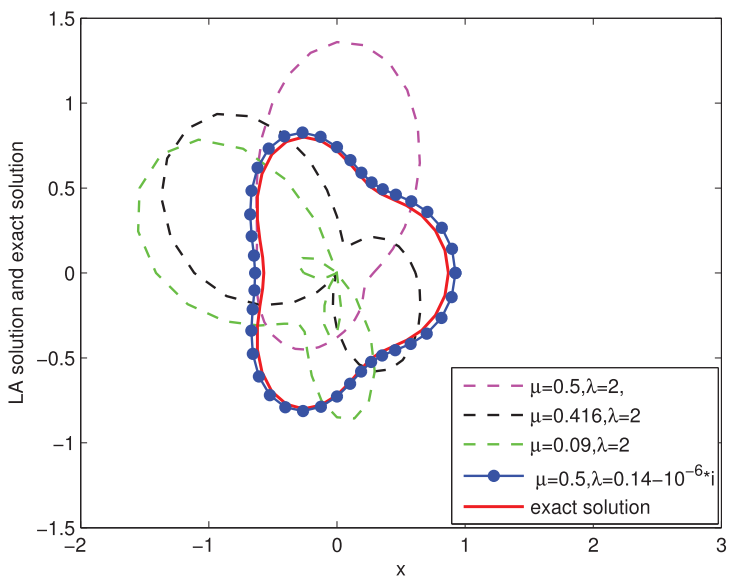

FiguRE 8. Reconstruction the shape for Example 16 with $5 \%$ noise data with various regularization parameter $\mu$.

TABLE 4. The relative errors for Example 16 with different regularization parameters $\mu$ and $\lambda$, under same noise level $\delta=0.05$.

\begin{tabular}{|c|c|c|c|c|c|c|c|c|c|c|c|}
\hline & \multicolumn{10}{|c|}{$\lambda=2$} & \multirow{2}{*}{$\begin{array}{c}\lambda=0.14-10^{-6} i \\
0.5\end{array}$} \\
\hline$\mu$ & 0.1 & 0.8 & 0.09 & 0.73 & 0.83 & 0.206 & 0.212 & 0.320 & 0.416 & 0.668 & \\
\hline$e_{\gamma}$ & 0.881 & 0.720 & 1.278 & 0.548 & 1.096 & 1.297 & 1.324 & 1.034 & 0.848 & 0.668 & 0.0625 \\
\hline
\end{tabular}

\section{Conclusions}

We investigate the inverse problem that utilizing the far-field measurement to reconstruct the shape of of an inclusion. The plasmon resonance is proposed to enhance the sensitivity of the reconstruction as well as to reduce the ill-posedness of the inverse problem. In fact, we derive the representation formula of shape sensitivity functional by using the asymptotic expansion method. Based on the asymptotic expansion of the eigenvalues and eigenfunctions of the Neumann-Poincaré operator, we further derive the delicate spectral representation of the shape sensitivity functional, which indicates that the sensitivity is improved greatly as the plasmon resonance occurs. Moreover, we combine the Tikhonov regularization method with the Laplace approximation to solve the inverse problem. This hybrid method not only calculates the minimizer accurately and quickly, but also captures the statistical information of the solution. Finally, extensive numerical experiments confirm our theoretical analysis and illustrate the promising and salient features of the proposed reconstruction scheme.

Acknowledgements. The work of M Ding and G Zheng were supported by the NSF of China (11301168) and NSF of Hunan (2020JJ4166) and NSF Innovation Platform Open Fund project of Hunan Province No. 20K030. The work of H Liu was supported by the Hong Kong RGC General Research Fund (projects 12302919, 12301218 and 11300821) and the NSFC/RGC Joint Research Grant (project N_CityU101/21).

\section{REFERENCES}

[1] H. Ammari, H. Kang, M. Lim and H. Zribi, The generalized polarization tensors for resolved imaging. Part I: Shape reconstruction of a conductivity inclusion. Math. Comp. 81 (2012) 367-386.

[2] H. Ammari, G. Ciraolo, H. Kang, H. Lee and G. Milton, Spectral theory of a Neumann-Poincaré-type operator and analysis of cloaking due to anomalous localized resonance. Arch. Ration. Mech. Anal. 208 (2013) 667-692. 
[3] H. Ammari, Y. Chow, K. Liu and J. Zou, Optimal shape design by partial spectral data. SIAM J. Sci. Comput. 37 (2015) B855-B883.

[4] H. Ammari, Y. Deng and P. Millien, Surface plasmon resonance of nanoparticles and applications in imaging. Arch. Ration. Mech. Anal. 220 (2016) 109-153.

[5] H. Ammari, P. Millien, M. Ruiz and H. Zhang, Mathematical analysis of plasmonic nanoparticles: the scalar case. Arch. Ration. Mech. Anal. 224 (2017) 597-658.

[6] H. Ammari, Y. Chow and H. Liu, Quantum ergodicity and localization of plasmon resonances. Preprint arXiv:2003.03696 (2020).

[7] H. Ammari, Y. Chow, H. Liu and M. Sunkula, Quantum integral systems and concentration of plasmon resonance. Preprint arXiv:2109.13008 (2021).

[8] H. Ammari, Y.T. Chow and H. Liu, Localized sensitivity analysis at high-curvature boundary points of reconstructing inclusions in transmission problems. SIAM J. Math. Anal. (2022) DOI: 10.1137/20M1323576.

[9] K. Ando, H. Kang and H. Liu, Plasmon resonance with finite frequencies: A validation of the quasi-static approximation for diametrically small inclusions. SIAM J. Appl. Math. 76 (2016) 731-749.

[10] J. Anker, W. Hall, O. Lyandres, N. Shah, J. Zhao and R. Van Duyne, Biosensing with plasmonic nanosensors, and Applications, Cambridge University Press, New York (2010).

[11] G. Baffou, C. Girard and R. Quidant, Mapping heat origin in plasmonic structures. Phys. Rev. Lett. 104 (2010) 136805.

[12] E. Blåsten, H. Li, H. Liu and Y. Wang, Localization and geometrization in plasmon resonances and geometric structures of Neumann-Poincaré eigenfunctions. ESAIM: M2AN 54 (2020) 957-976.

[13] E. Blåsten and H. Liu, Recovering piecewise constant refractive indices by a single far-field pattern. Inverse Probl. 36 (2020) 085005.

[14] E. Blåsten and H. Liu, On corners scattering stably and stable shape determination by a single far-field pattern. Indiana Univ. Math. J. 70 (2021) 907-947.

[15] E. Blåsten and H. Liu, Scattering by curvatures, radiationless sources, transmission eigenfunctions, and inverse scattering problems. SIAM J. Math. Anal. 53 (2021) 3801-3837.

[16] G. Bouchitté and B. Schweizer, Cloaking of small objects by anomalous localized resonance. Quart. J. Mech. Appl. Math. 63 (2010) 437-463.

[17] X. Cao, H. Diao, H. Liu and J. Zou, On nodal and generalized singular structures of Laplacian eigenfunctions and applications to inverse scattering problems. J. Math. Pures Appl. 143 (2020) 116-161.

[18] Y.T. Chow, Y. Deng, Y. He, H. Liu and X. Wang, Surface-localized transmission eigenstates, super-resolution imaging, and pseudo surface plasmon modes. SIAM J. Imaging Sci. 14 (2021) 946-975.

[19] A. Cintrón-Arias, H. Banks, A. Capaldi and A. Lloyd, A sensitivity matrix methodology for inverse problem formulation. J. Inverse Ill-Pose. P. 17 (2009) 1-20.

[20] Y. Deng, J. Li and H. Liu, On identifying magnetized anomalies using geomagnetic monitoring. Arch. Ration. Mech. Anal. 231 (2019) 153-187.

[21] Y. Deng, J. Li and H. Liu, On identifying magnetized anomalies using geomagnetic monitoring within a magnetohydrodynamic model. Arch. Ration. Mech. Anal. 235 (2020) 691-721.

[22] Y. Deng, H. Li and H. Liu, Analysis of surface polariton resonance for nanoparticles in elastic system. SIAM J. Math. Anal. 52 (2020) 1786-1805.

[23] Y. Deng, H. Liu and G. Zheng, Mathematical analysis of plasmon resonances for curved nanorods. J. Math. Pures Appl. 153 (2021) 248-280.

[24] Y. Deng, H. Liu and G. Zheng, Plasmon resonances of nanorods in transverse electromagnetic scattering. J. Differ. Eqs. 318 (2022) 502-536.

[25] H. Diao, X. Cao and H. Liu, On the geometric structures of transmission eigenfunctions with a conductive boundary condition and applications. Commun. Partial Differ. Equ. 46 (2021) 630-679.

[26] A. Doicu, T. Trautmann and F. Schreier, Numerical regularization for atmospheric inverse problems, Springer Science \& Business Media (2010).

[27] X. Fang, Y. Deng and J. Li, Plasmon resonance and heat generation in nanostructures. Math. Method. Appl. Sci. 38 (2015) 4663-4672.

[28] X. Fang, Y. Deng and H. Liu, Sharp estimate of electric field from a conductive rod and application. Stud. Appl. Math. 146 (2021) 279-297.

[29] T. Feng, H. Kang and H. Lee, Construction of GPT-vanishing structures using shape derivative. J. Comput. Math. 35 (2017) $569-585$.

[30] Y. Gao, H. Liu, X. Wang and K. Zhang, On an artificial neural network for inverse scattering problems. J. Comput. Phys. 448 (2022) 110771.

[31] D. Grieser, The plasmonic eigenvalue problem. Rev. Math. Phys. 26 (2014) 1450005.

[32] M. Hanke, A regularizing Levenberg-Marquardt scheme with applications to inverse groundwater filtration problems. Inverse Probl. 13 (1997) 79.

[33] M. Hanke, Recent progress in electrical impedance tomography. Inverse Probl. 19 (2003) S65-S90.

[34] M. Hintermüller, A. Laurain and I. Yousept, Shape sensitivities for an inverse problem in magnetic induction tomography based on the eddy current model. Inverse Probl. 31 (2015) 065006. 
[35] M. Iglesias, K. Law and A. Stuart, Evaluation of Gaussian approximations for data assimilation in reservoir models. Comput. Geosci. 17 (2013) 851-885.

[36] P. Jain, K. Lee, I. El-Sayed and M. El-Sayed, Calculated absorption and scattering properties of gold nanoparticles of different size, shape, and composition: applications in biomedical imaging and biomedicine. J. Phys. Chem. B 110 (2006) $7238-7248$.

[37] R. Kress, Linear Integral Equations, 2nd edition, Springer (1999).

[38] H. Li and H. Liu, On anomalous localized resonance and plasmonic cloaking beyond the quasistatic limit. Proc. Roy. Soc. A 474 (2018).

[39] H. Li, J. Li and H. Liu, On quasi-static cloaking due to anomalous localized resonance in $\mathbb{R}^{3}$. SIAM J. Appl. Math. 75 (2015) $1245-1260$.

[40] H. Li, J. Li and H. Liu, On novel elastic structures inducing polariton resonances with finite frequencies and cloaking due to anomalous localized resonances. J. Math. Pures Appl. 120 (2018) 195-219.

[41] H. Li, S. Li, H. Liu and X. Wang, Analysis of electromagnetic scattering from plasmonic inclusions beyond the quasi-static approximation and applications. ESAIM: M2AN 53 (2019) 1351-1371.

[42] S. Link and M. El-Sayed, Shape and size dependence of radiative, non-radiative and photothermal properties of gold nanocrystals. Int. Rev. Phys. Chem. 19 (2000) 409-453.

[43] H. Liu and C.H. Tsou, Stable determination of polygonal inclusions in Calderón's problem by a single partial boundary measurement. Inverse Probl. 36 (2020) 085010.

[44] H. Liu, C.H. Tsou and W. Yang, On Calderón's inverse inclusion problem with smooth shapes by a single partial boundary measurement. Inverse Probl. 37 (2021) 055005.

[45] I. Mayergoyz, D. Fredkin and Z. Zhang, Electrostatic (plasmon) resonances in nanoparticles. Phys. Rev. B 72 (2005) 155412.

[46] G. Milton and N. Nicorovici, On the cloaking effects associated with anomalous localized resonance. Proc. R. Soc. A 462 (2006) 3027-3059.

[47] J. Nam, C. Thaxton and C. Mirkin, Nanoparticle-based bio-bar codes for the ultrasensitive detection of proteins. Science 301 (2003) $1884-886$.

[48] D. Nicholls and X. Tong, A high-order perturbation of surfaces algorithm for the simulation of localized surface plasmon resonances in two dimensions. J. Sci. Comput. 76 (2018) 1370-1395.

[49] M. Ordal, L. Long, R. Bell, S. Bell, R. Bell, R. Alexander and C. Ward, Optical properties of the metals al, co, cu, au, fe, pb, ni, pd, pt, ag, ti, and w in the infrared and far infrared. Appl. Opt. 22 (1983) 1099-1119.

[50] G. Raschke, S. Kowarik, T. Franzl, C. Sönnichsen, T.A. Klar, J. Feldmann, A. Nichtl and K. Kürzinger, Biomolecular recognition based on single gold nanoparticle light scattering. Nano Lett. 3 (2003) 935-938.

[51] D. Sarid and W. Challener, Modern Introduction to Surface Plasmons: Theory, Mathematical Modeling. Nat. Mater. 7 (2008) $442-453$

[52] C. Schillings, B. Sprungk and P. Wacker, On the Convergence of the Laplace Approximation and Noise-Level-Robustness of Laplace-based Monte Carlo Methods for Bayesian Inverse Problems. Numer. Math 145 (2020) 915-971.

[53] S. Schultz, D. Smith, J. Mock and D. Schultz, Single-target molecule detection with nonbleaching multicolor optical immunolabels. Proc. Natl Acad. Sci. USA 97 (2000) 996-1001.

[54] C. Vogel, Computational Methods for Inverse Problems. SIAM (2002).

[55] W. Yin, W. Yang and H. Liu, A neural network scheme for recovering scattering obstacles with limited phaseless far-field data. J. Comput. Phys. 417 (2020) 109594.

[56] G. Zheng, Mathematical analysis of plasmonic resonance for 2-D photonic crystal. J. Differ. Eqs. 266 (2019) 5095-5117.

\section{Subscribe to Open (S2O) A fair and sustainable open access model}

This journal is currently published in open access under a Subscribe-to-Open model (S2O). S2O is a transformative model that aims to move subscription journals to open access. Open access is the free, immediate, online availability of research articles combined with the rights to use these articles fully in the digital environment. We are thankful to our subscribers and sponsors for making it possible to publish this journal in open access, free of charge for authors.

\section{Please help to maintain this journal in open access!}

Check that your library subscribes to the journal, or make a personal donation to the S2O programme, by contacting subscribers@edpsciences.org

More information, including a list of sponsors and a financial transparency report, available at: https://www. edpsciences.org/en/maths-s2o-programme 